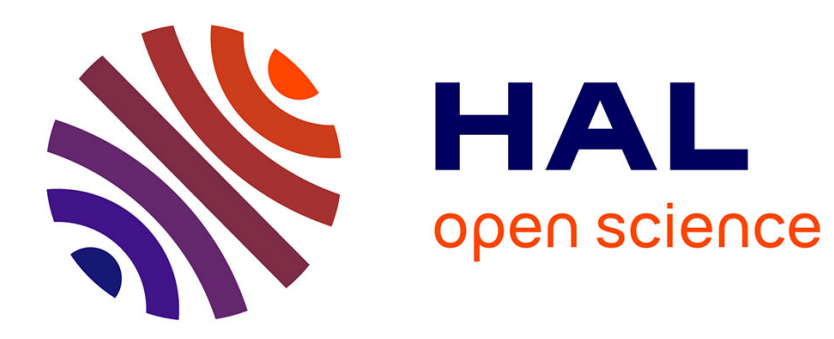

\title{
Display Calculi for Nominal Tense Logics
}

Stéphane Demri, Rajeev Goré

\section{To cite this version:}

Stéphane Demri, Rajeev Goré. Display Calculi for Nominal Tense Logics. Journal of Logic and Computation, 2002, 12 (6), pp.993-1016. 10.1093/logcom/12.6.993 . hal-03195079

\section{HAL Id: hal-03195079 \\ https://hal.science/hal-03195079}

Submitted on 14 Apr 2021

HAL is a multi-disciplinary open access archive for the deposit and dissemination of scientific research documents, whether they are published or not. The documents may come from teaching and research institutions in France or abroad, or from public or private research centers.
L'archive ouverte pluridisciplinaire HAL, est destinée au dépôt et à la diffusion de documents scientifiques de niveau recherche, publiés ou non, émanant des établissements d'enseignement et de recherche français ou étrangers, des laboratoires publics ou privés. 


\title{
Display Calculi for Nominal Tense Logics
}

\author{
Stéphane Demri \\ Lab. Spécification et Vérification \\ CNRS, UMR 8643 \\ ENS de Cachan \\ 61, av. du Pdt Wilson \\ 94235 Cachan Cedex, France \\ email: demri@lsv.ens-cachan.fr
}

\author{
Rajeev Goré \\ Automated Reasoning Group and \\ Department of Computer Science \\ Australian National University \\ Canberra ACT 0200, Australia \\ email: rpg@arp.anu.edu.au
}

\begin{abstract}
We define display calculi for nominal tense logics extending the minimal nominal tense logic (MNTL) by addition of primitive axioms. To do so, we use the natural translation of MNTL into the minimal tense logic of inequality $\left(\mathcal{L}_{\neq}\right)$which is known to be properly displayable by application of Kracht's results. The rules of the display calculus $\delta$ MNTL for MNTL mimic those of the display calculus $\delta \mathcal{L}_{\neq}$for $\mathcal{L}_{\neq}$. We show that every MNTL-valid formula admits a cut-free derivation in $\delta$ MNTL. We also show that a restricted display calculus $\delta^{-}$MNTL, is not only complete for MNTL, but that it enjoys cut-elimination for arbitrary sequents. Finally, we give a weak Sahlqvist-type theorem for two semantically defined extensions of MNTL. Using Kracht's techniques we obtain sound and complete display calculi for these two extensions based upon $\delta$ MNTL and $\delta^{-}$MNTL respectively. The display calculi based upon $\delta$ MNTL enjoy cut-elimination for valid formulae only, but those based upon $\delta^{-}$MNTL enjoy cut-elimination for arbitrary sequents.
\end{abstract}

\section{Introduction}

Background. The addition of names (also called nominals) to modal logics has been investigated recently with different motivations (see e.g. [Orło84, PT85, Gar86, Bla90]). A name is usually understood as an atomic proposition that holds true in a unique world of a Kripke model. Usually, the addition of names is intended to increase the expressive power of the initial logics. For instance, although there is no tense formula that characterises the class of irreflexive frames, there is a tense formula with names that characterises this class of frames [Bla93]. Another remarkable breakthrough due to the inclusion of names is the ability to define the intersection operator (see e.g. [PT91]) although it is known that intersection is not modally definable in the standard modal language [GT75]. Names have also been introduced in information logics [Orło84, Kon97b, Kon97a] derived from Pawlak's rough set theory [Paw81] where the motivations concern both definability and axiomatisability. Adding the difference operator $[\neq]$, which allows access to worlds different from the current world, is another way to obtain names (see e.g. [Sai88, Koy92, Rij92, Ven93]). As far as expressive power is concerned, adding $[\neq]$ is more powerful than adding 
only names: in [GG93], the relationships between names and $[\neq]$ are fully established with respect to definability. For instance, all universal first-order conditions on the Kripke reachability relation $\mathrm{R}$ (read as a binary predicate symbol) and $=$ (identity) are definable in the bimodal language with the standard necessity operator and the difference operator [Gor90]. In the literature for modal logics with names, much work has been dedicated to the study of their expressive power, decidability, complexity (see e.g. [Bla90, Rij93, GG93, PT91, ABM00]) and to the definition of proof systems [Bla90, PT91, Ven93, Rij93, Sel97, Dem99, Tza99, Are00].

Our contribution. Our main goal is to define cut-free display calculi (see e.g. [Bel82]) for nominal tense logics and therefore to provide a complementary approach to existing proof systems. In particular, we wish to extend previous results for displaying tense logics (see e.g. [Wan94, Kra96, Wan98]) to nominals. Display Logic (abbreviated by $\mathbf{D L}$ ) is a proof-theoretical framework introduced by Belnap [Bel82] that generalises the structural language of Gentzen's sequents by using multiple structural connectives instead of Gentzen's comma. A nice property of DL is its very general cut-elimination theorem [Bel82]. Furthermore, in the rules introducing logical connectives, the principal formula is alone as an antecedent or succedent thereby making the introduction rule a definition of that connective. In that sense, interactions between logical connectives are reduced to the minimum. This can be done since any occurrence of a structure in a sequent can be displayed either as the entire antecedent or as the entire succedent of some sequent "structurally equivalent" to the initial sequent.

So, the first contribution of the paper is the definition of display calculi for certain extensions of the minimal nominal tense logic (MNTL) [Bla90], by addition of primitive axioms in the sense of [Kra96]. Our first stage consists in defining the display calculus $\delta$ MNTL by using the natural translation from MNTL into $\mathrm{MTL}_{\neq}$, the minimal tense logic augmented with the difference operator. Indeed, $\mathrm{MTL}_{\neq}$is properly displayable ${ }^{1}$ in the sense of [Kra96] thanks to the Hilbert-style axiomatisation given in [Rij92, Rij93] (see also [Seg81, Koy92]). The powerful and sometimes redundant irreflexivity rule (see e.g. [Gab81, Ven93, Bal99]) is not needed to axiomatise MTL $\neq$. The rules for $\delta$ MNTL mimic those of $\delta \mathrm{MTL}_{\neq}$, the display calculus for MTL $\neq$. Completeness of $\delta$ MNTL is first proved by backward translation. The proof also shows that every MNTL-valid formula admits a cut-free derivation in $\delta$ MNTL. However, to extend $\delta$ MNTL, the above technique requires Hilbert-style axiomatisations that may contain the irreflexivity rule to appropriately extend $\mathrm{MTL}_{\neq}$. The corresponding rule in DL lacks various nice properties of the standard display calculi for tense logics. Furthermore, it is not always known when the irreflexivity rule is really needed. We therefore provide a second completeness proof of $\delta$ MNTL from the Hilbert-style calculus $\vdash_{\text {MNTL }}$ for MNTL given in [Bla90] for which the irreflexivity rule is never needed. The second proof happens to be much more informative since it provides a means to understand the rôle of various structural rules.

Cut-elimination cannot be proved by the technique of the second proof since it relies on the simulation of the modus ponens rule. An interesting, and at first glance very unpleasant, feature of $\delta$ MNTL is that it does not satisfy the condition (C8) [Bel82]. This condition is central in cut-elimination proofs from [Bel82, Wan98]. Nevertheless,

\footnotetext{
${ }^{1}$ This partly answers some open questions stated in [Wan98] and also suggests a general prooftheoretical framework for the $[\neq]$ operator (see also the questions in [Rij93, p.47]).
} 
we prove that a slight variant of $\delta$ MNTL admits cut-elimination for arbitrary sequents by Belnap's conditions while preserving completeness.

Finally, although many extensions of MNTL are not canonical [Bla90], we show a weak Sahlqvist-style theorem for nominal tense logics. This paves the way to define cut-free display calculi for any extension of $\vdash_{\text {MNTL }}$ by addition of primitive axioms (by using [Kra96]). Furthermore, we can characterise the semantical extensions of MNTL which correspond to these calculi.

Related work. Most of the proof systems for nominal tense logics are Hilbertstyle ones [Bla90, Bla93, Gor96a]. The situation is similar for modal logics with the difference operator [Seg81, Koy92, Rij92, Rij93, Ven93]. However, prefixed tableaux for several modal logics with the difference operator have been defined in [Dem96, BD97]. Decision procedures have been designed from these calculi [BD97] but a cut rule present in those calculi is not eliminable in many cases for reasons similar to those that apply to calculi from [dM94]. Sequent-style calculi for modal logics with names have been defined in [Kon97a, Kon97b] for the so-called similarity logics based on Pawlak's rough set theory [Paw81]. In [Kon97a, Kon97b], the nominals play the rôle of prefixes in an elegant manner, although the calculi have no prefixed formulae and only the language of the logic is used. In [Bla00], sequent calculi for nominal tense logics are given in which the nominals roughly play the role of labels; see also [Sel97, Dem99, Tza99, Are00].

Our treatment of nominals in our DL calculi is completely different since we instead use the dual nature of a nominal: as atomic proposition and as necessity formula. In that sense, it is similar to the treatment of atomic propositions in display calculi for intuitionistic logic in [Gor95].

A Sahlqvist theorem for tense logics with the difference operator is given in [Ven93], but the calculi use the irreflexivity rule; see also [Rij93].

Other general proof-theoretical frameworks also exist for non-classical logics: Labelled Deductive Systems [Gab96], Relational Proof Systems [Orło88, Orło91, Orło92] to quote two. But DL has already shown its generality since cut-free display calculi have been defined for substructural logics [Res98, Gor98b, Gor98a], for modal and polymodal logics [Wan94, Kra96, Wan98], for intuitionistic logics [Gor95], for relation algebras [Gor97, DG98], for logics with relative accessibility relations [DG00a] and for modal provability logics [DG00b, DG01b].

Plan of the paper. The rest of the paper is structured as follows. In Section 2, we recall the definitions of the logics under study [Bla90, Rij92, Ven93]. In Section 3, we introduce the modal logic of inequality $\mathrm{MTL}_{\neq}[\mathrm{Rij} 92]$ and formulate its (cut-free) display calculus $\delta \mathrm{MTL}_{\neq}$using the methodology of [Kra96]. In Section 4, we define the display calculus $\delta$ MNTL for MNTL, show its soundness and completeness, and prove it enjoys a weak form of cut-elimination. Section 5 contains a second independent completeness proof that easily extends to extensions of MNTL. In Section 6 , we show how cut-elimination can be obtained from Belnap's conditions for a slight variant of $\delta$ MNTL. Section 7 establishes a weak Sahlqvist-style theorem and by using [Kra96], defines cut-free display calculi for extensions of MNTL.

This paper is a completed and corrected version of [DG99]. 


\section{Nominal Tense Logics}

Given a set $\operatorname{Prp}=\left\{\mathrm{p}_{0}, \mathrm{p}_{1}, \mathrm{p}_{2}, \ldots\right\}$ of propositional variables and a set Nom $=$ $\left\{i_{0}, i_{1}, \ldots\right\}$ of names, the set $\operatorname{NTL}(G, H,[\neq])$ is the smallest set containing all formulae $\phi$ defined as below for all $\mathrm{p}_{j} \in \operatorname{Prp}$ and all $i_{k} \in$ Nom:

$\phi::=\top|\perp| \mathrm{p}_{j}\left|i_{k}\right| \phi_{1} \wedge \phi_{2}\left|\phi_{1} \vee \phi_{2}\right| \phi_{1} \Rightarrow \phi_{2}|\neg \phi| H \phi|G \phi|[\neq] \phi$.

Standard abbreviations include $\Leftrightarrow,\langle\neq\rangle, F, P$. For instance, $F \phi \stackrel{\text { def }}{=} \neg G \neg \phi$. Following standard notation, for any sequence $\overline{O P}$ from $\{H, G,[\neq]\}$ we write $\operatorname{NTL}(\overline{O P})$ to denote the fragment of $\operatorname{NTL}(G, H,[\neq])$ with the unary modal operators from $\overline{O P}$. Similarly, $\mathrm{TL}(\overline{O P})$ denotes the fragment of $\operatorname{NTL}(\overline{O P})$ with no names. In the rest of the paper, we shall study logics whose languages are strict fragments of $\operatorname{NTL}(G, H,[\neq])$ : the whole language contains all that we need. For any $\phi \in \operatorname{NTL}(G, H,[\neq])$, we write $r(\phi)$ to denote the rank of $\phi$; that is, the number of occurrences of members of

$$
\operatorname{Prp} \cup \operatorname{Nom} \cup\{\top, \perp\} \cup\{\neg, \wedge, \vee, \Rightarrow, G, H,[\neq]\} .
$$

For example $r\left(\perp \Rightarrow\left(i_{0} \vee \neg \mathrm{p}_{1}\right)\right)=6$.

Following [Kra96], a formula is primitive iff it is of the form $\phi \Rightarrow \psi$ where both $\phi$ and $\psi$ are built from $\operatorname{Prp} \cup\{\top\}$ with the help of $\wedge, \vee, F, P$ and $\langle\neq\rangle$ only, and $\phi$ contains each propositional variable at most once.

A modal frame $\mathcal{F}=(W, R)$ is a pair where $W$ is a non-empty set and $R$ is a binary relation over $W$. We write $F r$ for the set of all modal frames and define

$$
R(w) \stackrel{\text { def }}{=}\{v \in W \mid(w, v) \in R\} \quad R^{-1}(w) \stackrel{\text { def }}{=}\{v \in W \mid(v, w) \in R\} .
$$

A model $\mathcal{M}$ is a structure $\mathcal{M}=(W, R, m)$ such that $(W, R)$ is a frame and $m$ is a mapping $m: \operatorname{Prp} \cup$ Nom $\rightarrow \mathcal{P}(W)$ where for any $i \in$ Nom, $m(i)$ is a singleton, and where $\mathcal{P}(W)$ is the set of all subsets of $W$. Let $\mathcal{M}=(W, R, m)$ be a model and $w \in W$. As usual, the formula $\phi$ is satisfied by the world $w \in W$ in $\mathcal{M}$ iff $\mathcal{M}, w \models \phi$ where the satisfaction relation $\models$ is inductively defined as below:

$$
\begin{array}{cll}
\mathcal{M}, w \models \mathrm{p} & \text { if } & w \in m(\mathrm{p}), \text { for every } \mathrm{p} \in \operatorname{Prp} \cup \text { Nom } \\
\mathcal{M}, w \models G \phi & \text { if } & \mathcal{M}, v \models \phi \text { for every } v \in R(w) \\
\mathcal{M}, w \models H \phi & \text { if } & \mathcal{M}, v \models \phi \text { for every } v \in R^{-1}(w) \\
\mathcal{M}, w=[\neq] \phi & \text { if } & \mathcal{M}, v \models \phi \text { for every } v \neq w .
\end{array}
$$

We omit the standard conditions for the propositional connectives and the logical constants. A formula $\phi$ is true in a model $\mathcal{M}$ (written $\mathcal{M} \models \phi$ ) iff $\mathcal{M}, w \models \phi$ for every $w \in W$. A formula $\phi$ is true in a frame $\mathcal{F}$ (written $\mathcal{F}=\phi$ ) iff $\phi$ is true in every model based on $\mathcal{F}$.

By a logic $\mathcal{L}$ we mean a pair $\langle\mathrm{L}, \mathcal{C}\rangle$ such that $\mathrm{L} \subseteq \operatorname{NTL}(H, G,[\neq])$ and $\emptyset \neq \mathcal{C} \subseteq F r$. A formula $\phi \in \mathrm{L}$ is $\mathcal{L}$-valid iff $\phi$ is true in all the models based on the frames in $\mathcal{C}$. A formula $\phi \in \mathrm{L}$ is $\mathcal{L}$-satisfiable iff $\neg \phi$ is not $\mathcal{L}$-valid. A class $\mathcal{C}$ of modal frames is closed under disjoint unions iff for every $(W, R),\left(W^{\prime}, R^{\prime}\right) \in \mathcal{C}$ : if $W \cap W^{\prime}=\emptyset$ then $\left(W \cup W^{\prime}, R \cup R^{\prime}\right) \in \mathcal{C}$. A class $\mathcal{C}$ of modal frames is closed under isomorphic copies iff for any $(W, R) \in \mathcal{C}$ and for any 1-1 mapping $g: W \rightarrow W^{\prime}$, the frame $\left(W^{\prime},\left\{\left\langle g(w), g\left(w^{\prime}\right)\right\rangle \mid\left\langle w, w^{\prime}\right\rangle \in R\right\}\right) \in \mathcal{C}$. 
We write MNTL to denote the minimal nominal tense logic

$$
\text { MNTL } \stackrel{\text { def }}{=}\langle\operatorname{NTL}(H, G), F r\rangle .
$$

Moreover, for any formula $\phi$ of some language $\mathrm{L}$ with names [resp. without names], we write $\mathrm{NTL}_{\phi}\left[\right.$ resp. $\left.\mathrm{TL}_{\phi}\right]$ to denote the logic $\langle\mathrm{L},\{\mathcal{F} \in F r: \mathcal{F} \models \phi\}\rangle$.

To conclude this section, we recall the definitions of various Hilbert-style systems for the minimal nominal tense logic from [Bla90]. By a universal modality [resp. existential modality] $\sigma$, we mean a (possibly empty) finite sequence of elements from $\{G, H\}$ [resp. from $\{F, P\}]$. Let $\vdash_{\text {MNTL }}$ be the smallest subset of NTL $(G, H)$ such that $\vdash_{\text {MNTL }}$ is closed under the inference rules of modus ponens and necessitation for $G$ and $H ; \vdash_{\text {MNTL }}$ contains the tautologies of the propositional calculus; and $\vdash_{\text {MNTL }}$ contains every formula of the forms below:

$$
\begin{array}{ll}
(G(\phi \Rightarrow \psi) \wedge G \phi) \Rightarrow G \psi & (H(\phi \Rightarrow \psi) \wedge H \phi) \Rightarrow H \psi \\
\phi \Rightarrow H F \phi & \phi \Rightarrow G P \phi \\
i \wedge \phi \Rightarrow \sigma(i \Rightarrow \phi) \text { where } i \in \text { Nom and } \sigma \text { is a universal modality. }
\end{array}
$$

We write $\vdash_{\text {MNTL }} \phi$ or $\phi \in \vdash_{\text {MNTL }}$ interchangeably. Let $\vdash_{\text {MNTL }}^{\prime}$ be $\vdash_{\text {MNTL }}$ with the last axiom schema replaced by $i \wedge \sigma(i \wedge \phi) \Rightarrow \phi$ where $\sigma$ is an existential modality.

Theorem 1 Any $\phi \in \operatorname{NTL}(H, G)$ is MNTL-valid iff $\vdash_{\text {MNTL }} \phi$ iff $\vdash_{\text {MNTL }}^{\prime} \phi$ [Bla90].

We write $\vdash+\phi$ to denote the minimal extension of the axiomatic system $\vdash$ by adding all formulae of the form $\phi$; so $\phi$ is just an axiom schema.

\section{A Logic Axiomatised by Primitive Axioms}

We now give a Hilbert-style calculus for the minimal tense logic of inequality $\mathrm{MTL}_{\neq}$and a cut-free display calculus $\delta \mathrm{MTL}_{\neq}$for it using Kracht's method [Kra96].

\subsection{Hilbert-style Axiomatisation for $\mathrm{MTL}_{\neq}$}

In [Rij93], a complete Hilbert-style proof system is given for the logic

$$
\mathrm{MTL}_{\neq} \stackrel{\text { def }}{=}\langle\mathrm{TL}(G, H,[\neq]), F r\rangle .
$$

Let $\vdash_{\neq}$be the smallest set of $\operatorname{TL}(G, H,[\neq])$ containing the tautologies of the propositional calculus such that $\vdash_{\neq}$is closed under modus ponens, necessitation for $G, H$ and $[\neq]$, and where $\vdash_{\neq}$contains every formula of the forms:

$$
\begin{array}{llc}
(G(\phi \Rightarrow \psi) \wedge G \phi) \Rightarrow G \psi & \phi \Rightarrow H F \phi & F \phi \Rightarrow \phi \vee\langle\neq\rangle \phi \\
(H(\phi \Rightarrow \psi) \wedge H \phi) \Rightarrow H \psi & \phi \Rightarrow G P \phi & P \phi \Rightarrow \phi \vee\langle\neq\rangle \phi \\
([\neq](\phi \Rightarrow \psi) \wedge[\neq] \phi) \Rightarrow[\neq] \psi & \phi \Rightarrow[\neq]\langle\neq\rangle \phi & \langle\neq\rangle\langle\neq\rangle \phi \Rightarrow \phi \vee\langle\neq\rangle \phi .
\end{array}
$$

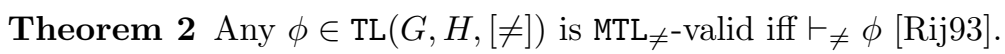




$$
\text { (Id) } \mathrm{p} \vdash \mathrm{p} \quad \frac{\mathrm{X} \vdash \phi \quad \phi \vdash \mathrm{Y}}{\mathrm{X} \vdash \mathrm{Y}} \text { (cut) }
$$

Figure 1: Fundamental logical axioms and cut rule

\subsection{A Display Calculus for $\mathrm{MTL}_{\neq}$}

There are numerous existing display calculi so we extend Wansing's [Wan94] formulation since it is tailored for classical modal logics. To follow Kracht's methodology [Kra96], MTL $\neq$ must be axiomatised by a set of primitive axioms. To do so, we replace the non-primitive axiom $\phi \Rightarrow[\neq]\langle\neq\rangle \phi$ by its primitive equivalent $\phi \wedge\langle\neq\rangle \psi \Rightarrow\langle\neq\rangle(\psi \wedge\langle\neq\rangle \phi)$. By applying [Kra96, Theorem 21], MTL $\neq$ can be "properly displayed": that is, MTL $\neq$ has a sound and complete display calculus $\delta \mathrm{MTL}_{\neq}$for which the cut-elimination theorem holds because $\delta \mathrm{MTL}_{\neq}$satisfies Belnap's conditions (C1)(C8) [Bel82]. Moreover, axioms from the definition of $\vdash_{\neq}$are encoded in $\delta \mathrm{MTL}_{\neq}$by structural rules: rules that involve only structure variables. In the rest of this section, we explicitly formulate the display calculus $\delta \mathrm{MTL}_{\neq}$for $\mathrm{MTL}_{\neq}$obtained by application of Kracht's results. We also use this opportunity to introduce smoothly various notions and to state basic facts that are used to define the display calculi for nominal tense logics. Hence, this section is mainly included to make the paper self-contained.

On the structural side, we have the structural connectives $*$ (unary), o (binary), $I$ (nullary), $\bullet$ (unary) and $\bullet \neq($ unary $)$. A structure $\mathrm{X} \in \operatorname{struc}\left(\delta \mathrm{MTL}_{\neq}\right)$is inductively defined as below for $\phi \in \operatorname{TL}(G, H,[\neq])$ :

$$
\mathrm{X}::=\phi|I| * \mathrm{X}|\bullet \mathrm{X}| \bullet \neq \mathrm{X} \mid \mathrm{X}_{1} \circ \mathrm{X}_{2}
$$

A sequent is an expression $\mathrm{X} \vdash \mathrm{Y}$, built from two structures $\mathrm{X}$ and $\mathrm{Y}$, with $\mathrm{X}$ the antecedent and $\mathrm{Y}$ the succedent. Figures $1-5$ contain the rules of $\delta \mathrm{MTL}_{\neq}$.

The display postulates (reversible rules) in Figure 2 deal with the manipulation of structural connectives. In what follows, we write

$$
\frac{s^{\prime}}{s}(d p)
$$

to denote that the sequent $s$ is obtained from the sequent $s^{\prime}$ by an unspecified finite number (possibly zero) of applications of display postulates.

In any structure $\mathrm{X}$, the structure Z occurs negatively [resp. positively] iff Z occurs in the scope of an odd number [resp. an even number] of occurrences of $*$ [Bel82]. In a sequent $\mathrm{X} \vdash \mathrm{Y}$, an occurrence of $\mathrm{Z}$ is an antecedent part [resp. succedent part] iff it occurs positively in $X$ [resp. negatively in $Y$ ] or it occurs negatively in $Y$ [resp. positively in $\mathrm{X}$ ] [Bel82]. Two sequents $\mathrm{X} \vdash \mathrm{Y}$ and $\mathrm{X}^{\prime} \vdash \mathrm{Y}^{\prime}$ are structurally equivalent iff there is a derivation of the first sequent from the second using the display postulates from Figure 2.

Theorem 3 (Belnap) For every sequent $\mathrm{X} \vdash \mathrm{Y}$ and every antecedent [resp. succedent] part $\mathrm{Z}$ of $\mathrm{X} \vdash \mathrm{Y}$, there is a structurally equivalent sequent $\mathrm{Z} \vdash \mathrm{Y}^{\prime}\left[\right.$ resp. $\left.\mathrm{X}^{\prime} \vdash \mathrm{Z}\right]$ that has 


\begin{tabular}{|c|c|c|c|c|c|}
\hline & $\mathrm{X} \circ \mathrm{Y} \vdash \mathrm{Z}$ & $\mathrm{X} \circ \mathrm{Y} \vdash \mathrm{Z}$ & $X \vdash Y \circ Z$ & \multicolumn{2}{|c|}{$\mathrm{X} \vdash \mathrm{Y} \circ \mathrm{Z}$} \\
\hline & $\mathrm{X} \vdash \mathrm{Z} \circ * \mathrm{Y}$ & $\mathrm{Y} \vdash * \mathrm{X} \circ \mathrm{Z}$ & $\mathrm{X} \circ * \mathrm{Z} \vdash \mathrm{Y}$ & \multicolumn{2}{|c|}{$* \mathrm{Y} \circ \mathrm{X} \vdash \mathrm{Z}$} \\
\hline$* \mathrm{X} \vdash \mathrm{Y}$ & $\mathrm{X} \vdash * \mathrm{Y}$ & $* * \mathrm{X} \vdash \mathrm{Y}$ & $\mathrm{X} \vdash * * \mathrm{Y}$ & $\mathrm{X} \vdash \bullet \neq \mathrm{Y}$ & $\mathrm{X} \vdash \bullet \mathrm{Y}$ \\
\hline$* \mathrm{Y} \vdash \mathrm{X}$ & $\mathrm{Y} \vdash * \mathrm{X}$ & $\mathrm{X} \vdash \mathrm{Y}$ & $\mathrm{X} \vdash \mathrm{Y}$ & $\bullet \neq \mathrm{X} \vdash \mathrm{Y}$ & $\bullet \mathrm{X} \vdash \mathrm{Y}$ \\
\hline
\end{tabular}

Figure 2: Display postulates

$$
\begin{aligned}
& \overline{I \vdash \top}(\vdash \top) \quad \frac{I \vdash \mathrm{X}}{\top \vdash \mathrm{X}}(\top \vdash) \quad \frac{\mathrm{X} \vdash I}{\mathrm{X} \vdash \perp}(\vdash \perp) \quad \frac{}{\perp \vdash I}(\perp \vdash) \\
& \frac{\mathrm{X} \vdash * \phi}{\mathrm{X} \vdash \neg \phi}(\vdash \neg) \quad \frac{* \phi \vdash \mathrm{X}}{\neg \phi \vdash \mathrm{X}}(\neg \vdash) \frac{\mathrm{X} \vdash \phi \mathrm{Y} \vdash \psi}{\mathrm{X} \circ \mathrm{Y} \vdash \phi \wedge \psi}(\vdash \wedge) \quad \frac{\phi \circ \psi \vdash \mathrm{X}}{\phi \wedge \psi \vdash \mathrm{X}}(\wedge \vdash) \\
& \frac{\mathrm{X} \circ \phi \vdash \psi}{\mathrm{X} \vdash \phi \Rightarrow \psi}(\vdash \Rightarrow) \frac{\mathrm{X} \vdash \phi \psi \vdash \mathrm{Y}}{\phi \Rightarrow \psi \vdash * \mathrm{X} \circ \mathrm{Y}}(\Rightarrow \vdash) \\
& \frac{\mathrm{X} \vdash \phi \circ \psi}{\mathrm{X} \vdash \phi \vee \psi}(\vdash \vee) \frac{\phi \vdash \mathrm{X} \psi \vdash \mathrm{Y}}{\phi \vee \psi \vdash \mathrm{X} \circ \mathrm{Y}}(\vee \vdash) \frac{\phi \vdash \mathrm{X}}{G \phi \vdash \bullet \mathrm{X}}(G \vdash) \frac{\mathrm{X} \vdash \bullet \phi}{\mathrm{X} \vdash G \phi}(\vdash G) \\
& \frac{\phi \vdash \mathrm{X}}{H \phi \vdash * \bullet * \mathrm{X}}(H \vdash) \frac{\mathrm{X} \vdash * \bullet * \phi}{\mathrm{X} \vdash H \phi}(\vdash H) \quad \frac{\phi \vdash \mathrm{X}}{[\neq] \phi \vdash \bullet \neq \mathrm{X}}([\neq] \vdash) \frac{\mathrm{X} \vdash \bullet \neq \phi}{\mathrm{X} \vdash[\neq] \phi}(\vdash[\neq])
\end{aligned}
$$

Figure 3: Operational rules

$\mathrm{Z}$ (alone) as its antecedent [resp. succedent]. The structure $\mathrm{Z}$ is displayed in $\mathrm{Z} \vdash \mathrm{Y}^{\prime}$ [resp. $\left.\mathrm{X}^{\prime} \vdash \mathrm{Z}\right][$ Bel82].

In Figure 5, "alio" stands for aliotransitivity where a binary relation $R$ over $W$ is aliotransitive iff for any $x, y, z \in W,(x, y) \in R$ and $(y, z) \in R$ and $x \neq z$ implies $(x, z) \in R$ (see e.g. [Seg81]). The structural rules defined in Figure 5 are translations of the primitive axioms of $\vdash_{\neq}$into structural rules following [Kra96]. This is done modulo the rule (sym) as shown below.

Lemma 4 Let $\mathrm{X} \vdash \mathrm{Y}$ and $\mathrm{X}^{\prime} \vdash \mathrm{Y}^{\prime}$ be sequents such that $\mathrm{X}^{\prime} \vdash \mathrm{Y}^{\prime}$ can be obtained from $\mathrm{X} \vdash \mathrm{Y}$ by replacing some occurrences of $* \bullet \neq * \mathrm{Z}$ by $\bullet \neq \mathrm{Z}$ and by replacing some occurrences of $\bullet \neq \mathrm{Z}^{\prime}$ by $* \bullet * \mathrm{Z}^{\prime}$. Then, in any display calculus $\delta$ containing the display postulates from Figure 2, $($ sym $),\left(\right.$ contr $\left._{r}\right),\left(w e a k_{r}\right)$ and $\left(w e a k_{l}\right)$, the sequent $\mathrm{X} \vdash \mathrm{Y}$ is [cut-free] derivable in $\delta$ iff $\mathrm{X}^{\prime} \vdash \mathrm{Y}^{\prime}$ is [cut-free] derivable in $\delta$.

Proof Thanks to the display property, it is sufficient to show:

1. If $* \bullet \neq * \mathrm{Z} \vdash \mathrm{X}$ is [cut-free] derivable in $\delta$, then so is $\bullet \neq \mathrm{Z} \vdash \mathrm{X}$. 


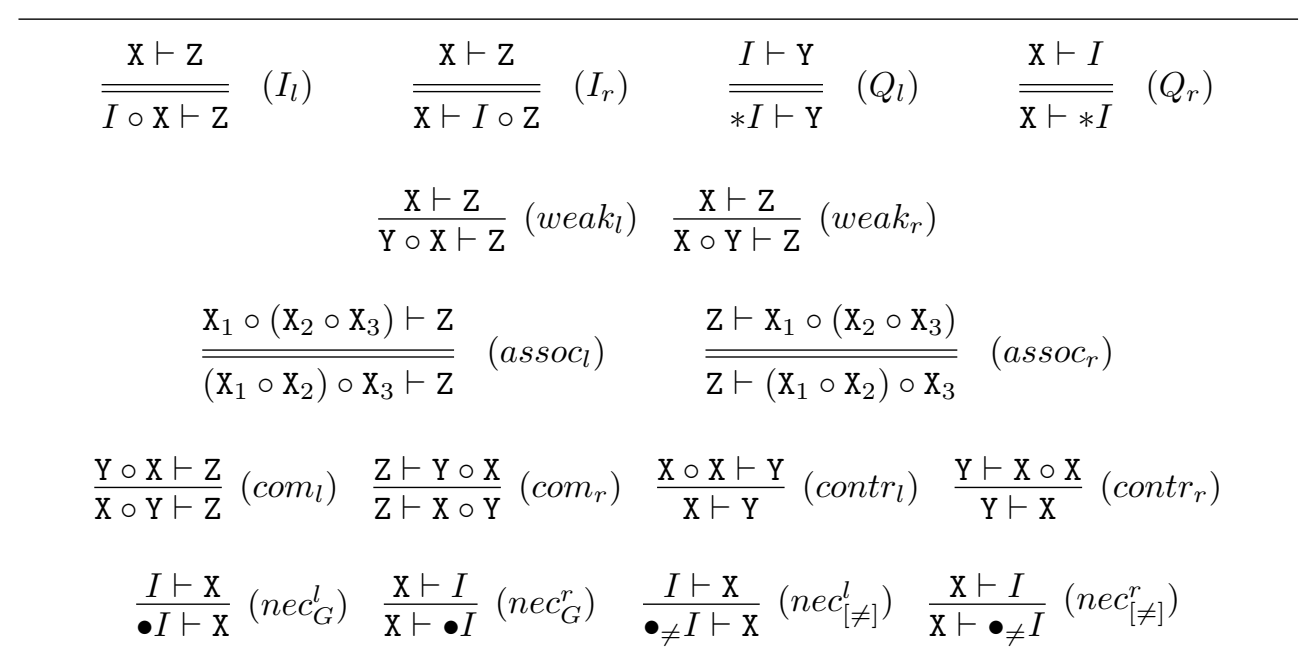

Figure 4: Other basic structural rules

$$
\begin{aligned}
& \frac{\mathrm{X} \vdash \mathrm{Y} \quad \bullet \neq \mathrm{X} \vdash \mathrm{Y}}{\bullet \neq \bullet \neq \mathrm{X} \vdash \mathrm{Y}} \text { (alio) } \quad \frac{* \bullet_{\neq} *(\mathrm{Z} \circ * \bullet \neq * \mathrm{X}) \vdash \mathrm{Y}}{\mathrm{X} \circ * \bullet \neq * \mathrm{Z} \vdash \mathrm{Y}} \text { (sym) } \\
& \frac{\mathrm{X} \vdash \mathrm{Y} \quad \bullet \neq \mathrm{X} \vdash \mathrm{Y}}{* \bullet * \mathrm{X} \vdash \mathrm{Y}}(\text { uni } 1) \quad \frac{\mathrm{X} \vdash \mathrm{Y} \quad \bullet \neq \mathrm{X} \vdash \mathrm{Y}}{\bullet \mathrm{X} \vdash \mathrm{Y}}(\text { uni } 2)
\end{aligned}
$$

Figure 5: Other structural rules

2. If $\bullet \neq \mathrm{Z} \vdash \mathrm{X}$ is [cut-free] derivable in $\delta$, then so is $* \bullet \neq * \mathrm{Z} \vdash \mathrm{X}$.

Remember also that the following are derivable using the display postulates:

$$
\frac{* \bullet \neq * \mathrm{Z} \vdash \mathrm{X}}{\mathrm{Z} \vdash * \bullet \neq * \mathrm{X}}(d p) \quad \frac{\bullet \neq \mathrm{Z} \vdash \mathrm{X}}{\mathrm{Z} \vdash \bullet_{\neq} \mathrm{X}}(d p)
$$

We show (1) below left and (2) below right:
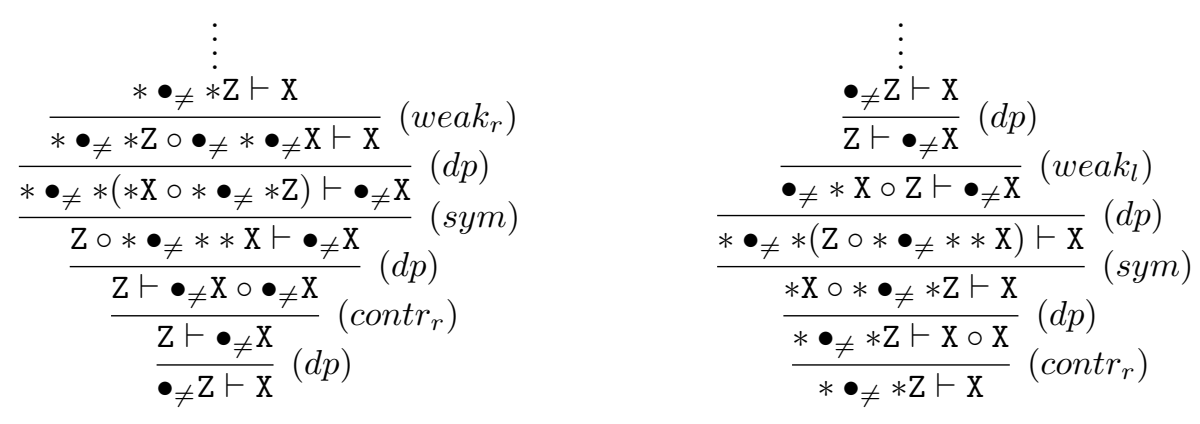
In Lemma 4 we can replace the rule $(s y m)$ by the rule $\left(s y m^{\prime}\right)$ shown below left. Lemma 4 is unsurprising since (sym) is the structural rule obtained from Kracht's methodology from the axiom schema $F \phi \Leftrightarrow P \phi$ characterising symmetric models. But, Lemma 4 is purely syntactic since it contains no reference to any interpretation of the structures. We make extensive use of the derivable invertible rule (sym) shown below right which encapsulates Lemma 4:

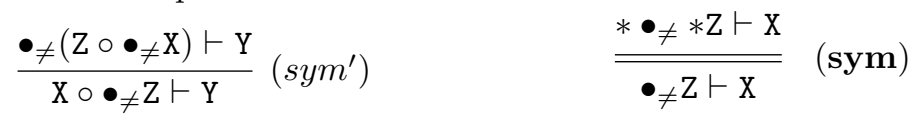

We abuse notation since we also use (sym) for the rule that consists of replacing any occurrence of $\bullet \neq$ [resp. $* \bullet * *$ in a sequent by $* \bullet \neq *$ [resp. $\bullet$ ] by displaying the "target", applying (sym), and then "undisplaying". Such inferences may require the "right-handed" analogues of our structural rules, which are left unspecified, but which are derivable. The correct form of these "right-handed" analogues is not always the rule obtained by simply switching the antecedents and conclusions of the sequents in the "left-handed" version.

Primitivity of the axioms guarantees a sound and complete display calculus satisfying conditions (C1)-(C8) [Bel82] and therefore enjoying cut-elimination.

Theorem 5 (Soundness, Completeness) For any formula $\phi \in \operatorname{NTL}(G, H,[\neq])$, the sequent $I \vdash \phi$ is derivable in $\delta \mathrm{MTL}_{\neq}$iff $\vdash_{\neq} \phi[\mathrm{Kra96}]$.

Theorem 6 (Cut-elimination) If there is a derivation of $\mathrm{X} \vdash \mathrm{Y}$ in $\delta \mathrm{MTL}_{\neq}$, then there is a cut-free derivation of $\mathrm{X} \vdash \mathrm{Y}$ in $\delta \mathrm{MTL} \neq[$ Bel82].

We write $\delta+\mathcal{R}$ for display calculus $\delta$ augmented with the set $\mathcal{R}$ of rules.

\section{A Display Calculus for MNTL}

We use a standard embedding of MNTL into $\mathrm{MTL}_{\neq} \neq$to obtain a display calculus $\delta$ MNTL from the display calculus $\delta \mathrm{MTL}_{\neq}$. We prove soundness and completeness of $\delta$ MNTL with respect to MNTL by using the soundness and completeness of $\delta \mathrm{MTL}_{\neq}$with respect to $\mathrm{MTL}_{\neq}$. We also prove a weak cut-elimination theorem for $\delta$ MNTL by using the cutelimination theorem for $\delta \mathrm{MTL}_{\neq}$. Actually, we show that for every $\phi \in \operatorname{NTL}(H, G), I \vdash \phi$ is derivable in $\delta$ MNTL iff $I \vdash \phi$ has a cut-free derivation in $\delta$ MNTL. "Weak" because cut-elimination is couched using arbitrary sequents $\mathrm{X} \vdash \mathrm{Y}$ rather than sequents of the form $I \vdash \phi$. In Section 5 we then prove completeness of $\delta$ MNTL directly with respect to the Hilbert-style calculus $\vdash_{\text {mNTL }}$.

\subsection{Definition of $\delta$ MNTL}

The display calculus $\delta$ MNTL defined below is composed of axioms and inference rules from $\delta \mathrm{MTL}_{\neq}$defined in Section 3.2. The set $\operatorname{struc}(\delta \mathrm{MNTL})$ of structures is the same as $\operatorname{struc}\left(\delta \operatorname{MTL}_{\neq}\right)$except that only formulae from $\operatorname{NTL}(H, G)$ can occur as substructures although the structural connectives are identical. The fundamental logical axiom and cut rule (Figure 1), the structural rules (Figures 2, 4 and 5) and the operational rules (Figure 3 ) for $\delta$ MNTL are by definition those for $\delta \mathrm{MTL}_{\neq}$except that the rules 


$$
\frac{i \vdash \bullet * \mathrm{X}}{\mathrm{X} \vdash i}(\vdash i) \quad \frac{\mathrm{X} \vdash i}{i \vdash \bullet \neq * \mathrm{X}}(i \vdash) \quad\left(I d^{\prime}\right) i \vdash i
$$

Figure 6: Axioms and rules for $\delta$ MNTL

introducing $[\neq] \phi$, as antecedent and succedent, are replaced by the rules introducing the nominals, as antecedent and succedent, described in Figure 6. The fundamental axiom $\left(I d^{\prime}\right)$ for nominals is also added.

An easy way to understand the way the rules in Figure 6 work is to observe that the formula from $\operatorname{NTL}([\neq])$ shown below is valid in any model:

$$
i \Leftrightarrow[\neq] \neg i .
$$

The rules $(i \vdash)$ and $(\vdash i)$ use the intensional nature of a name whereas the fundamental axiom $i \vdash i$ uses its atomic nature.

Using the display postulates and the derived rule (sym), we can alternatively define the "introduction" rules for nominals as follows:

$$
\frac{\mathrm{x} \vdash \bullet * i}{\mathrm{X} \vdash i} \quad \frac{\mathrm{X} \vdash i}{\mathrm{X} \vdash \bullet \neq * i}
$$

Following [Kra96], it is easy to establish that both rules $(* \vdash)$ and $(\vdash *)$ below are admissible in $\delta$ MNTL:

$$
\frac{\neg \psi \vdash \mathrm{X}}{* \psi \vdash \mathrm{X}}(* \vdash) \quad \frac{\mathrm{X} \vdash \neg \psi}{\mathrm{X} \vdash * \psi}(\vdash *)
$$

In particular, if $\neg \psi \vdash \mathrm{X}$ [resp. $\mathrm{X} \vdash \neg \psi$ ] has a cut-free derivation in $\delta$ MNTL, then $* \psi \vdash \mathrm{X}$ $[$ resp. $\mathrm{X} \vdash * \psi$ ] also has a cut-free derivation in $\delta$ MNTL.

\subsection{Soundness and Completeness}

To prove soundness of $\delta$ MNTL with respect to MNTL-validity we use the mappings $a$ : $\operatorname{struc}(\delta \mathrm{MNTL}) \rightarrow \mathrm{TL}(H, G,[\neq])$ and $c: \operatorname{struc}(\delta \mathrm{MNTL}) \rightarrow \mathrm{TL}(H, G,[\neq])$ defined below

\begin{tabular}{|c|c|c|c|c|c|}
\hline$a\left(i_{k}\right)$ & $\stackrel{\text { def }}{=}$ & $\mathrm{p}_{2 \times k+1} \wedge[\neq] \neg \mathrm{p}_{2 \times k+1}$ & $c\left(i_{k}\right)$ & $\stackrel{\text { def }}{=}$ & $\mathrm{p}_{2 \times k+1} \wedge[\neq] \neg \mathrm{p}_{2 \times k+1}$ \\
\hline$a\left(\mathrm{p}_{j}\right)$ & $\stackrel{\text { def }}{=}$ & $\mathrm{p}_{2 \times j}$ & $c\left(\mathrm{p}_{j}\right)$ & $\stackrel{\text { def }}{=}$ & $\mathrm{p}_{2 \times j}$ \\
\hline$a(I)$ & $\stackrel{\text { def }}{=}$ & $T^{\circ}$ & $c(I)$ & $\stackrel{\text { def }}{=}$ & $\perp$ \\
\hline$a(\perp)$ & $\stackrel{\text { def }}{=}$ & $\perp$ & $c(\perp)$ & $\stackrel{\text { def }}{=}$ & $\perp$ \\
\hline$a(\top)$ & $\stackrel{\text { def }}{=}$ & $T$ & $c(\top)$ & $\stackrel{\text { def }}{=}$ & $\top$ \\
\hline$a(* \mathrm{X})$ & $\stackrel{\text { def }}{=}$ & $\neg c(\mathrm{X})$ & $c(* \mathrm{X})$ & $\stackrel{\text { def }}{=}$ & $\neg a(\mathrm{X})$ \\
\hline$a(\mathrm{X} \circ \mathrm{Y})$ & $\stackrel{\text { def }}{=}$ & $a(\mathrm{X}) \wedge a(\mathrm{Y})$ & $c(\mathrm{X} \circ \mathrm{Y})$ & & $c(\mathrm{X}) \vee c(\mathrm{Y})$ \\
\hline$a(\bullet \mathrm{X})$ & $\stackrel{\text { def }}{=}$ & $P a(\mathrm{X})$ & $c(\bullet \mathbf{X})$ & $\stackrel{\text { def }}{=}$ & $G c(\mathrm{X})$ \\
\hline$a(\bullet \neq \mathrm{X})$ & $\stackrel{\text { def }}{=}$ & $\langle\neq\rangle a(\mathrm{X})$ & $c(\bullet \neq \mathrm{X})$ & $\stackrel{\text { def }}{=}$ & {$[\neq] c(\mathrm{X})$.} \\
\hline
\end{tabular}
which are are slight variants of standard mappings; see e. g. [Kra96]:

$a$ and $c$ are homomorphic for $\wedge, \vee, \Rightarrow, \neg, H$ and $G$

$$
i_{k} \in \text { Nom } \quad \mathrm{p}_{j} \in \operatorname{Prp}
$$


For any finite set $S$ of structures, we write $\operatorname{Nom}(S)$ for the set of names from Nom that occur in $S$. We also define the formula $\phi_{S}$ from $\operatorname{TL}(G, H,[\neq])$ below:

$$
\varphi_{S} \stackrel{\text { def }}{=} \bigwedge_{i_{k} \in \operatorname{Nom}(S)}\left(\mathrm{p}_{2 \times k+1} \wedge[\neq] \neg \mathrm{p}_{2 \times k+1}\right) \vee\langle\neq\rangle\left(\mathrm{p}_{2 \times k+1} \wedge[\neq] \neg \mathrm{p}_{2 \times k+1}\right) .
$$

In the case when $\operatorname{Nom}(S)$ is empty, $\varphi_{S}$ takes the value $T$. For any finite set $S$ and for any MTL $_{\neq}$-model $\mathcal{M}=(W, R, m), \mathcal{M}=\varphi_{S}$ iff $\mathcal{M}, w=\varphi_{S}$ for some $w \in W$.

Lemma 7 easily follows from the definition of the formulae of the form $\varphi_{S}$.

Lemma 7 Let $S, S^{\prime}$ be finite sets of structures, $\psi$ be in $\operatorname{NTL}(G, H)$ and $\psi^{\prime}$ be in $\mathrm{TL}(G, H,[\neq])$. Then,

1. if $S^{\prime} \subseteq S$, then $\varphi_{S} \Rightarrow \varphi_{S^{\prime}}$ is $\mathrm{MTL}_{\neq}$-valid;

2. if for all $k \in \omega$, the propositional variable $\mathrm{p}_{2 \times k+1}$ occurs in $\psi^{\prime}$ only if $i_{k}$ occurs in $S$, then $\varphi_{S} \Rightarrow \psi^{\prime}$ is $\operatorname{MTL}_{\neq}$-valid iff $\varphi_{S \cup\{\psi\}} \Rightarrow \psi^{\prime}$ is $\mathrm{MTL}_{\neq}$-valid.

Lemma 8 below relates the formulae of the form $\varphi_{S}$ with the map $a($.$) .$

Lemma 8 For some class of frames $\mathcal{C}$, let $\mathcal{L}=\langle\operatorname{NTL}(G, H), \mathcal{C}\rangle$ be the nominal tense logic of $\mathcal{C}$-frames and let $\mathcal{L}_{\neq}=\langle\mathrm{TL}(G, H,[\neq]), \mathcal{C}\rangle$ be the tense logic of inequality of $\mathcal{C}$-frames. Then, for any $\phi \in \operatorname{NTL}(G, H)$, statements (1) and (2) below are equivalent:
(1) $\phi$ is $\mathcal{L}$-valid
(2) $\varphi_{\{\phi\}} \Rightarrow a(\phi)$ is $\mathcal{L}_{\neq^{-} \text {valid. }}$

Since $a(\neg \phi)=\neg a(\phi)$, it suffices to prove that statements (3) and (4) below are equivalent:
(3) $\phi$ is $\mathcal{L}$-satisfiable
(4) $\varphi_{\{\phi\}} \wedge a(\phi)$ is $\mathcal{L}_{\neq \text {-satisfiable. }}$

Proof $(\mathbf{3}) \Rightarrow(4)$ : Suppose $\phi$ is $\mathcal{L}$-satisfiable. So there is an $\mathcal{L}$-model $\mathcal{M}=(W, R, m)$ and $w_{0} \in W$ such that $\mathcal{M}, w_{0} \models \phi$. Let $\mathcal{M}^{\prime}=\left(W, R, m^{\prime}\right)$ be the $\mathcal{L}_{\neq}$-model such that for all $k \in \omega$ :

$$
m^{\prime}\left(\mathrm{p}_{2 \times k}\right) \stackrel{\text { def }}{=} m\left(\mathrm{p}_{k}\right) \quad m^{\prime}\left(\mathrm{p}_{2 \times k+1}\right) \stackrel{\text { def }}{=} m\left(i_{k}\right) .
$$

For $w \in W$ and for $k \in \omega$ it is obvious that:

$$
\begin{aligned}
& \mathcal{M}, w \models \mathrm{p}_{k} \text { iff } \mathcal{M}^{\prime}, w \models \mathrm{p}_{2 \times k} \\
& \mathcal{M}, w \models i_{k} \text { iff } \mathcal{M}^{\prime}, w \models \mathrm{p}_{2 \times k+1} \wedge[\neq] \neg \mathrm{p}_{2 \times k+1} \\
& \mathcal{M}^{\prime}, w \models \varphi_{\{\phi\}} .
\end{aligned}
$$

These are the base cases to show by induction on the structure of any subformula $\psi$ of $\phi$ that for any $w \in W: \mathcal{M}, w \models \psi$ iff $\mathcal{M}^{\prime}, w \models a(\psi)$. Hence, $\mathcal{M}^{\prime}, w_{0} \models \varphi_{\{\phi\}} \wedge a(\phi)$.

(4) $\Rightarrow(3)$ : Suppose $\varphi_{\{\phi\}} \wedge a(\phi)$ is $\mathcal{L}_{\neq \text {-satisfiable. }}$ So there is an $\mathcal{L}_{\neq^{-} \text {-model }} \mathcal{M}=$ $(W, R, m)$ and $w_{0} \in W$ such that $\mathcal{M}, w_{0} \models \varphi_{\{\phi\}} \wedge a(\phi)$. Let $\mathcal{M}^{\prime}=\left(W, R, m^{\prime}\right)$ be the $\mathcal{L}$-model such that for every $k \in \omega$ : 


$$
\begin{aligned}
& m^{\prime}\left(\mathrm{p}_{k}\right) \stackrel{\text { def }}{=} m\left(\mathrm{p}_{2 \times k}\right) \\
& m^{\prime}\left(i_{k}\right)=\{x\} \text { if } i_{k} \text { occurs in } \phi \text { and } \mathcal{M}, x \models \mathrm{p}_{2 \times k+1} \wedge[\neq] \neg \mathrm{p}_{2 \times k+1} \\
& m^{\prime}\left(i_{k}\right)=\left\{w_{0}\right\} \text { (arbitrary value) if } i_{k} \text { does not occur in } \phi .
\end{aligned}
$$

In the second clause above, a unique such $x \in W$ always exists because $\mathcal{M}, w_{0} \models$ $\varphi_{\{\phi\}}$. For $w \in W$ and for $k \in \omega$ it is obvious that:

$$
\mathcal{M}^{\prime}, w=\mathrm{p}_{k} \text { iff } \mathcal{M}, w \models \mathrm{p}_{2 \times k}
$$

if $i_{k}$ occurs in $\phi$ then: $\mathcal{M}^{\prime}, w \models i_{k}$ iff $\mathcal{M}, w \models \mathrm{p}_{2 \times k+1} \wedge[\neq] \neg \mathrm{p}_{2 \times k+1}$.

These are the base cases to show by induction on the structure of any subformula $\psi$ of $\phi$ that for any $w \in W: \mathcal{M}^{\prime}, w \models \psi$ iff $\mathcal{M}, w \models a(\psi)$. Hence, $\mathcal{M}^{\prime}, w_{0} \models \phi$.

Lemma 9 below allows us to get rid of $\varphi_{\{\phi\}}$ in $\varphi_{\{\phi\}} \Rightarrow a(\phi)$.

Lemma 9 For some class of frames $\mathcal{C}$ which is closed under disjoint unions and isomorphic copies, let $\mathcal{L}=\langle\operatorname{NTL}(G, H), \mathcal{C}\rangle$ be the nominal tense logic of $\mathcal{C}$-frames, and let $\mathcal{L}_{\neq}=\langle\mathrm{TL}(G, H,[\neq]), \mathcal{C}\rangle$ be the tense logic of inequality of $\mathcal{C}$-frames. For every $\phi \in \operatorname{NTL}(G, H)$, the statements (1) and (2) below are equivalent:

(1) $\phi$ is $\mathcal{L}$-valid $\quad(2) a(\phi)$ is $\mathcal{L}_{\neq \text {-valid. }}$

Since $a(\neg \phi)=\neg a(\phi)$, it suffices to prove that the statements (3) and (4) below are equivalent:

(3) $\phi$ is $\mathcal{L}$-satisfiable $\quad(4) a(\phi)$ is $\mathcal{L}_{\neq \text {-satisfiable. }}$

Proof $(3) \Rightarrow(4)$ : Similar to the part $(3) \Rightarrow(4)$ in the proof of Lemma 8 .

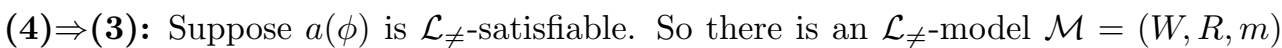
and $w_{0} \in W$ such that $\mathcal{M}, w_{0}=a(\phi)$. Here, we cannot guarantee that for any $i_{k}$ occurring in $\phi$, the set $U_{k}=\left\{x \in W \mid \mathcal{M}, x \models \mathrm{p}_{2 \times k+1} \wedge[\neq] \neg \mathrm{p}_{2 \times k+1}\right\}$ is a singleton.

However, to see that $U_{k}$ is a singleton assume that there are $w_{1} \neq w_{2} \in W$ such that $\left\{w_{1}, w_{2}\right\} \subseteq U_{k}$. Since $\mathcal{M}, w_{1} \models \mathrm{p}_{2 \times k+1} \wedge[\neq] \neg \mathrm{p}_{2 \times k+1}$ and $w_{1} \neq w_{2}$, we must have $\mathcal{M}, w_{2} \models \neg \mathrm{p}_{2 \times k+1}$, contradicting the definition of $U_{k}$. So, for any $i_{k}$ occurring in $\phi$, either $U_{k}=\emptyset$ or $U_{k}$ is a singleton.

Let $\mathcal{M}^{\prime}=\left(W^{\prime}, R^{\prime}, m^{\prime}\right)$ be the triple such that:

1. $W^{\prime}=W \times\{1,2\}$

2. $\langle w, j\rangle R^{\prime}\left\langle w^{\prime}, j^{\prime}\right\rangle \stackrel{\text { def }}{\Leftrightarrow} j=j^{\prime}$ and $w R w^{\prime}$

3. $m^{\prime}\left(\mathrm{p}_{k}\right) \stackrel{\text { def }}{=}\left\{\langle w, j\rangle \mid w \in m\left(\mathrm{p}_{2 \times k}\right), j \in\{1,2\}\right\}$ for every $k \in \omega$

4. for every $k \in \omega$ such that $i_{k}$ occurs in $\phi$,

$$
m^{\prime}\left(i_{k}\right)= \begin{cases}\{\langle x, 1\rangle\} & \text { if } U_{k} \neq \emptyset \text { and } \mathcal{M}, x \models \mathrm{p}_{2 \times k+1} \wedge[\neq] \neg \mathrm{p}_{2 \times k+1} \\ \left\{\left\langle w_{0}, 2\right\rangle\right\} & \text { if } U_{k}=\emptyset .\end{cases}
$$

5. for $k \in \omega$ where $i_{k}$ does not occur in $\phi, m^{\prime}\left(i_{k}\right)=\left\{\left\langle w_{0}, 2\right\rangle\right\}$. 
In clause 4 , such a unique $x \in W$ is guaranteed to exist and in clause 5 , the world $\left\langle w_{0}, 2\right\rangle$ is just an arbitrary value from the second copy of $\mathcal{M}$.

Since $\mathcal{C}$ is closed under isomorphic copies and disjoint unions, $\left(W^{\prime}, R^{\prime}\right) \in \mathcal{C}$ and $\mathcal{M}^{\prime}$ is an $\mathcal{L}$-model since the nominals are interpreted as singletons. For $w \in W$ and for $k \in \omega$ it is obvious that:

$$
\begin{aligned}
& \mathcal{M}^{\prime},\langle w, 1\rangle \models \mathrm{p}_{k} \text { iff } \mathcal{M}, w \models \mathrm{p}_{2 \times k} \\
& \text { if } i_{k} \text { occurs in } \phi \text {, then: } \\
& \qquad \mathcal{M}^{\prime},\langle w, 1\rangle \models i_{k} \text { iff } \mathcal{M}, w \models \mathrm{p}_{2 \times k+1} \wedge[\neq] \neg \mathrm{p}_{2 \times k+1} .
\end{aligned}
$$

These are the base cases to show by induction on the structure of any subformula $\psi$ of $\phi$ that for any $w \in W: \mathcal{M}^{\prime},\langle w, 1\rangle=\psi$ iff $\mathcal{M}, w \mid=a(\psi)$. Hence, $\mathcal{M}^{\prime},\left\langle w_{0}, 1\right\rangle \models \phi$.

Theorem 10 If $\mathrm{X} \vdash \mathrm{Y}$ is derivable in $\delta$ MNTL, then $\varphi_{\{\mathrm{X}, \mathrm{Y}\}} \Rightarrow(a(\mathrm{X}) \Rightarrow c(\mathrm{Y}))$ is $\mathrm{MTL}_{\neq^{-}}$ valid.

Proof The proof is by induction on the length of the given derivation of $\mathrm{X} \vdash \mathrm{Y}$. The base case with instances of $(I d)$ and $\left(I d^{\prime}\right)$ and $(\vdash \top)$ and $(\perp \vdash)$ are immediate. Assume that the theorem holds for all $\delta$ MNTL derivations of length less than some natural number $n>0$, and consider a derivation of length $n$. We now consider the bottom-most rule application in this derivation.

Cut Rule: Assume $\varphi_{\{\mathrm{X}, \phi\}} \Rightarrow(a(\mathrm{X}) \Rightarrow c(\phi))$ and $\varphi_{\{\mathrm{Y}, \phi\}} \Rightarrow(a(\phi) \Rightarrow c(\mathrm{Y}))$ are $\mathrm{MTL}_{\neq^{-}}$ valid. By Lemma $7(1), \varphi_{\{\mathrm{X}, \mathrm{Y}, \phi\}} \Rightarrow(a(\mathrm{X}) \Rightarrow c(\phi))$ and $\varphi_{\{\mathrm{X}, \mathrm{Y}, \phi\}} \Rightarrow(a(\phi) \Rightarrow c(\mathrm{Y}))$ are also $\mathrm{MTL}_{\neq}$-valid. Since $a(\phi)=c(\phi)$, we obtain $\varphi_{\{\mathrm{X}, \mathrm{Y}, \phi\}} \Rightarrow(a(\mathrm{X}) \Rightarrow c(\mathrm{Y}))$ is $\mathrm{MTL}_{\neq- \text {-valid. By Lemma }} 7(2), \varphi_{\{\mathrm{X}, \mathrm{Y}\}} \Rightarrow(a(\mathrm{X}) \Rightarrow c(\mathrm{Y}))$ is also $\mathrm{MTL}_{\neq}$-valid.

Rule $\frac{\mathrm{X} \vdash \bullet \mathrm{Y}}{\overline{\bullet \mathrm{X} \vdash \mathrm{Y}}} \quad$ Let us consider the proof for the direction from top to bottom. Suppose $\varphi_{\{\mathrm{X}, \mathrm{Y}\}} \Rightarrow(a(\mathrm{X}) \Rightarrow G c(\mathrm{Y}))$ is $\mathrm{MTL}_{\neq}$-valid and suppose $\varphi_{\{\mathrm{X}, \mathrm{Y}\}} \Rightarrow(\mathrm{Pa}(\mathrm{X}) \Rightarrow$ $c(\mathrm{Y}))$ is not $\mathrm{MTL}_{\neq}$-valid. There exist an $\mathrm{MTL}_{\neq}$-model $(W, R, m)$ and $w \in W$ such that $\mathcal{M}, w \models \varphi_{\{\mathrm{X}, \mathrm{Y}\}} \wedge P a(\mathrm{X}) \wedge \neg c(\mathrm{Y})$. So there is $w^{\prime} \in R^{-1}(w)$ such that $\mathcal{M}, w^{\prime} \models a(\mathrm{X})$, and $\mathcal{M}, w^{\prime} \models \varphi_{\{\mathrm{X}, \mathrm{Y}\}}$. By supposition, $\mathcal{M}, w^{\prime} \models G c(\mathrm{Y})$ and hence $\mathcal{M}, w \models c(\mathrm{Y})$, a contradiction.

$(\vdash i)$-rule: Assume $\varphi_{\left\{i_{k}, \mathrm{x}\right\}} \Rightarrow\left(\left(\mathrm{p}_{2 \times k+1} \wedge[\neq] \neg \mathrm{p}_{2 \times k+1}\right) \Rightarrow[\neq] \neg a(\mathrm{X})\right)$ is $\mathrm{MTL}_{\neq \neq}$-valid and suppose $\varphi_{\left\{i_{k}, \mathrm{X}\right\}} \Rightarrow\left(a(\mathrm{X}) \Rightarrow\left(\mathrm{p}_{2 \times k+1} \wedge[\neq] \neg \mathrm{p}_{2 \times k+1}\right)\right)$ is not $\mathrm{MTL}_{\neq}$-valid. Thus there exists an MTL $\neq$-model $(W, R, m)$ and $w \in W$ such that $\mathcal{M}, w \models \varphi_{\left\{i_{k}, \mathrm{x}\right\}} \wedge$ $a(\mathrm{X}) \wedge \neg\left(\mathrm{p}_{2 \times k+1} \wedge[\neq] \neg \mathrm{p}_{2 \times k+1}\right)$. Let $w^{\prime}$ be the unique element of $W$ such that $\mathcal{M}, w^{\prime}=\left(\mathrm{p}_{2 \times k+1} \wedge[\neq] \neg \mathrm{p}_{2 \times k+1}\right)$. Such an element $w^{\prime} \in W$ exists and is unique since $\mathcal{M} \models \varphi_{\left\{i_{k}, \mathrm{x}\right\}}$. Hence, $\mathcal{M}, w^{\prime} \models \varphi_{\left\{i_{k}, \mathrm{x}\right\}} \wedge\left(\mathrm{p}_{2 \times k+1} \wedge[\neq] \neg \mathrm{p}_{2 \times k+1}\right)$. Obviously $w^{\prime} \neq w$ and by supposition, $\mathcal{M}, w^{\prime} \models[\neq] \neg a(\mathrm{X})$. Hence, $\mathcal{M}, w \models \neg a(\mathrm{X})$, a contradiction.

$\left(\right.$ nec $\left._{[\neq]}^{l}\right)$-rule: Assume $\varphi_{\{\mathrm{x}\}} \Rightarrow(a(I) \Rightarrow c(\mathrm{X}))$ is $\mathrm{MTL}_{\neq}$-valid and suppose that $\varphi_{\{\mathrm{x}\}} \Rightarrow$ $(\langle\neq\rangle a(I) \Rightarrow c(\mathrm{X}))$ is not $\mathrm{MTL}_{\neq}$-valid. There exists an $\mathrm{MTL}_{\neq}$-model $(W, R, m)$ and $w \in W$ such that $\mathcal{M}, w \models \varphi_{\{\mathrm{x}\}} \wedge\langle\neq\rangle \top \wedge \neg c(\mathrm{X})$. By assumption, $\varphi_{\{\mathrm{x}\}} \Rightarrow c(\mathrm{X})$ is MTL $_{\neq}$-valid, so $\mathcal{M}, w \models c(\mathrm{X})$, a contradiction. 
(uni1): Assume (i) $\varphi_{\{\mathrm{X}, \mathrm{Y}\}} \Rightarrow(a(\mathrm{X}) \Rightarrow c(\mathrm{Y}))$ and (ii) $\varphi_{\{\mathrm{X}, \mathrm{Y}\}} \Rightarrow(\langle\neq\rangle a(\mathrm{X}) \Rightarrow c(\mathrm{Y}))$ are $\mathrm{MTL}_{\neq}$-valid. Suppose $\varphi_{\{\mathrm{X}, \mathrm{Y}\}} \Rightarrow(F a(\mathrm{X}) \Rightarrow c(\mathrm{Y}))$ is not $\mathrm{MTL}_{\neq}$-valid. There exists an $\operatorname{MTL}_{\neq}$-model $(W, R, m)$ and $w \in W$ such that $\mathcal{M}, w \models \varphi_{\{\mathrm{X}, \mathrm{Y}\}} \wedge F a(\mathrm{X}) \wedge \neg c(\mathrm{Y})$. So, there is $w^{\prime} \in R(w)$ such that $\mathcal{M}, w^{\prime} \models \varphi_{\{\mathrm{X}, \mathrm{Y}\}} \wedge a(\mathrm{X})$. By validity of (i), $\mathcal{M}, w^{\prime} \models c(\mathrm{Y})$. So $w \neq w^{\prime}$. That is, there is $w^{\prime} \neq w$ such that $\mathcal{M}, w^{\prime} \models$ $a(\mathrm{X})$. Hence, $\mathcal{M}, w \models \varphi_{\{\mathrm{X}, \mathrm{Y}\}} \wedge\langle\neq\rangle a(\mathrm{X})$. By validity of (ii), $\mathcal{M}, w \models c(\mathrm{Y})$, a contradiction.

Corollary 11 (Soundness) If $I \vdash \phi$ is derivable in $\delta$ MNTL, then $\phi$ is MNTL-valid.

Proof It is sufficient to observe that any formulae $\phi \in \mathrm{NTL}(H, G)$ is MNTL-valid iff $a(\phi)$ is $\mathrm{MTL}_{\neq}$-valid iff $\varphi_{\{\phi\}} \Rightarrow a(\phi)$ is $\mathrm{MTL}_{\neq}$-valid.

To prove completeness, we define a partial function

$$
g: \operatorname{struc}\left(\delta \mathrm{MTL}_{\neq}\right) \rightarrow \operatorname{struc}(\delta \operatorname{MNTL})
$$

as follows:

$g(\mathrm{X})$ is undefined if $\mathrm{X}$ contains any formula occurrence $[\neq] \psi$ with $\psi$ not of the form $\neg \mathrm{p}_{2 \times k+1}$ for any $k \in \omega$; otherwise

$g$ is homomorphic for the Boolean connectives, for $H$ and for $G$

$g\left(\mathrm{p}_{2 \times k+1}\right) \stackrel{\text { def }}{=} g\left([\neq] \neg \mathrm{p}_{2 \times k+1}\right) \stackrel{\text { def }}{=} i_{k}$ for any $k \in \omega$

$g\left(\mathrm{p}_{2 \times j}\right) \stackrel{\text { def }}{=} \mathrm{p}_{j}$ for any $j \in \omega$

$$
g(\perp) \stackrel{\text { def }}{=} \perp \quad g(\top) \stackrel{\text { def }}{=} \top \quad g(I) \stackrel{\text { def }}{=} I
$$

$g$ is homomorphic for the structural connectives.

It is easy to check that for any formula $\phi \in \operatorname{NTL}(H, G)$ the formula $g(a(\phi))$ is MNTL-valid iff $\phi$ is MNTL-valid. The following lemmata are used later.

Lemma 12 Suppose formulae $\phi^{\prime}$ from $\operatorname{NTL}(H, G)$ is obtained from $\phi$ by replacing some occurrences of the subformula $\psi$ of $\phi$ by $\psi \wedge \psi$. Then, $I \vdash \phi$ has a cut-free derivation in $\delta$ MNTL iff $I \vdash \phi^{\prime}$ has a cut-free derivation in $\delta$ MNTL.

Lemma 13 For any formula $\phi$ from $\operatorname{NTL}(H, G)$, the sequent $I \vdash \phi$ has a cut-free derivation in $\delta$ MNTL iff $I \vdash g(a(\phi))$ has a cut-free derivation in $\delta$ MNTL.

Proof It is a routine task to check that $g(a(\phi))$ can be obtained from $\phi$ by replacing every occurrence of a name $i_{k}$ by $i_{k} \wedge i_{k}$. By Lemma 12, the desired result follows. The extra copy is introduced by the translation $a($.$) .$

Theorem 14 (Completeness and Weak Cut-elimination) If a formula $\phi$ from the language $\operatorname{NTL}(H, G)$ is MNTL-valid, then $I \vdash \phi$ has a cut-free derivation in $\delta$ MNTL.

We denote that the sequent $s$ has a derivation $\Pi$ as shown below:

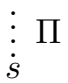


Proof Let $\phi$ be a formula of $\operatorname{NTL}(G, H)$. We must show that if $\phi$ is MNTL-valid, then $I \vdash \phi$ has a cut-free derivation in $\delta$ MNTL. There are five parts to the proof:

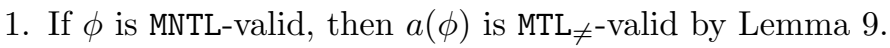

2. If $a(\phi)$ is $\mathrm{MTL}_{\neq}$-valid, then $I \vdash a(\phi)$ has a cut-free derivation in $\delta \mathrm{MTL}_{\neq}$by Theorem 5 and Theorem 6.

3. If $I \vdash a(\phi)$ has a cut-free derivation in $\delta \mathrm{MTL}_{\neq}$, then $I \vdash g(a(\phi))$ has a cut-free derivation in $\delta$ MNTL.

4. $I \vdash g(a(\phi))$ has a cut-free derivation in $\delta$ MNTL iff $I \vdash \phi$ has a cut-free derivation in $\delta$ MNTL by Lemma 13.

5. Hence, if $\phi$ is MNTL-valid, then $I \vdash \phi$ has a cut-free derivation in $\delta$ MNTL.

It remains to prove point (3). We show that in the given cut-free derivation of $I \vdash a(\phi)$, for every sequent $\mathrm{X} \vdash \mathrm{Y}$ with cut-free derivation $\Pi$, the sequent $g(\mathrm{X}) \vdash g(\mathrm{Y})$ admits a cut-free derivation, say $g(\Pi)$, in $\delta$ MNTL. It is worth observing that thanks to the subformula property of $\delta \mathrm{MTL}_{\neq}, g(\mathrm{X})$ and $g(\mathrm{Y})$ are always defined.

The proof is by induction on the length of the derivations. When $\mathrm{X} \vdash \mathrm{Y}$ is a fundamental logical axiom, or an instance of $(\vdash T)$ or $(\perp \vdash)$, the base case is immediate. Similarly, the proof poses no difficulty when the last rule is a structural rule from Figure 2, Figure 4 and Figure 5, or an operational rule introducing a Boolean connective, $H$ or $G$ since $g$ is homomorphic for the Boolean connectives, $H, G$ and the structural connectives.

Now let us treat the case when the last rule is $(\vdash[\neq])$. The derivation shown below left is transformed into the derivation shown below right:

$$
\begin{aligned}
& \vdots g(\Pi)
\end{aligned}
$$

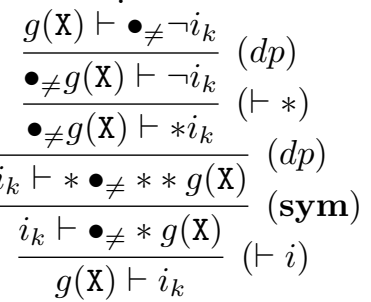

Observe that the inference $(\vdash *)$ in the above derivation is correct because by induction hypothesis, $g(\Pi)$ is cut-free; see the proof of admissibility of $(\vdash *)$ and $(* \vdash)$ in [Kra96] which requires cut-free derivations. Note that the proofs from [Gor96b] which derive $(\vdash *)$ and $(* \vdash)$ rather than show them admissible are not applicable here, since they assume cut-elimination holds.

Similarly, let us treat the case when the last rule is $([\neq] \vdash)$. The derivation shown below left is transformed into the derivation shown below right:

$$
\begin{gathered}
\vdots g(\Pi) \\
\frac{\neg i_{k} \vdash g(\mathrm{X})}{* i_{k} \vdash g(\mathrm{X})}(* \vdash) \\
\frac{\frac{* g(\mathrm{X}) \vdash i_{k}}{i_{k} \vdash \bullet * * * g(\mathrm{X})}}{\frac{i_{k} \vdash \bullet}{i_{k} \vdash(\mathrm{X})}}(d \vdash) \\
(d p)
\end{gathered}
$$


Corollary 15 For any formula $\phi \in \operatorname{NTL}(H, G)$, if $\Pi$ is a cut-free derivation of $I \vdash a(\phi)$ in $\delta \mathrm{MTL}_{\neq}$, then $I \vdash \phi$ has a cut-free derivation $\Pi^{\prime}$ in $\delta$ MNTL of size $\mathcal{O}(|\Pi|)$.

The proof of Corollary 15 also relies on the fact that if $\neg \psi \vdash \mathrm{X}$ [resp. X $\vdash \neg \psi]$ has a cut-free derivation $\Pi$ in $\delta$ MNTL, then $* \psi \vdash \mathrm{X}$ [resp. $\mathrm{X} \vdash * \psi]$ has a cut-free derivation $\Pi^{\prime}$ of size $\mathcal{O}(|\Pi|)$ in $\delta$ MNTL.

The rules $(i \vdash)$ and $(\vdash i)$ are obviously equivalent to the reversible rule below:

$$
\overline{\overline{\mathrm{X} \vdash i}}
$$

As for the display postulates, or for any reversible rule, naive backward applications of such rules may lead to loops in proof search. As a corollary of the proof of Theorem 14, if we apply $(i \vdash)$ [resp. $(\vdash i)$ ] upwards, we never need to apply $(\vdash i)$ [resp. $(i \vdash)$ ] to the name "introduced" into the premiss by these rules. Thus rules $(i \vdash)$ and $(\vdash i)$ themselves need not lead to loops in proof search.

The natural translation (restriction of $a$ ) from MNTL into $\mathrm{MTL}_{\neq}$has led to a relatively easy proof of Theorem 14 using strong existing results from the literature. However, extending this technique to extensions of MNTL by using the appropriate extensions of $\mathrm{MTL}_{\neq}$is problematic because we cannot guarantee that these latter extensions are axiomatisable using a set of primitive axioms. In particular, it is even an open problem to know when the irreflexivity rule is needed (see e.g. [Ven93]). Furthermore, the cut-elimination result of Theorem 14 is restricted to sequents of the form $I \vdash \phi$, rather than the more general $\mathrm{X} \vdash$ Y. But cut-elimination for such general sequents is not straightforward since $\delta$ MNTL does not satisfy Belnap's condition (C8) (see Section 6).

\section{Another Completeness Proof for $\delta$ MNTL}

Although Corollary 15 states very satisfactory results, the proofs in Section 4 cannot be easily generalised. In particular, for various extensions of $\vdash \neq$ where the irreflexivity rule shown below is added (see e.g. [Gab81, Rij92, Ven93]):

$$
\frac{\mathrm{p} \wedge[\neq] \neg \mathrm{p} \Rightarrow \phi}{\phi} \quad(\mathrm{IRR}) \text { provided propositional variable } \mathrm{p} \text { does not occur in } \phi
$$

It is unlikely that there is a natural DL rule corresponding to (IRR) that satisfies the conditions (C1)-(C8) [Bel82]. In the rest of this section, we give a completeness proof of $\delta$ MNTL based on the system $\vdash_{\text {MNTL }}$ [Bla90] whose extensions do not require the irreflexivity rule.

Lemma $16 I \vdash \phi_{1} \wedge \phi_{2} \Rightarrow \phi_{3}$ is [cut-free] derivable in $\delta$ MNTL iff $\phi_{1} \circ \phi_{2} \vdash \phi_{3}$ is [cut-free] derivable in $\delta$ MNTL.

Proof First we derive $I \vdash \phi_{1} \wedge \phi_{2} \Rightarrow \phi_{3}$ from $\phi_{1} \circ \phi_{2} \vdash \phi_{3}$ by using the rules $(\vdash \Rightarrow)$, $\left(w_{e a k_{l}}\right)$ and $(\wedge \vdash)$. Second, by proving that the rule below is admissible (without introducing new cuts), as in the proof of [Kra96, Lemma 9], we get the desired result:

$$
\frac{\mathrm{X} \vdash \phi \Rightarrow \psi}{\mathrm{X} \circ \phi \vdash \psi}
$$


Theorem 17 (Completeness) If $\vdash_{\text {MNTL }} \phi$, then $I \vdash \phi$ is derivable in $\delta$ MNTL.

Proof The proof is by induction on the length of the derivation of $\vdash_{\text {mNTL }} \phi$. Actually, most of the cases have been already proved in [Wan94, Kra96, Wan98]. It remains to show that

$$
I \vdash i \wedge \phi \Rightarrow \sigma(i \Rightarrow \phi)
$$

is derivable in $\delta$ MNTL where $i \in \operatorname{Nom}, \phi \in \operatorname{NTL}(H, G)$ and $\sigma$ is a (possibly empty) finite sequence of elements from $\{H, G\}$. To do so, we prove by induction on the length of $\sigma$ that both
(1) $i \circ \phi \vdash \sigma(i \Rightarrow \phi)$
and
$(2) \bullet \neq(i \circ \phi) \vdash \sigma(i \Rightarrow \phi)$

are derivable in $\delta$ MNTL, also doing some extra work that will prove useful later. By Lemma 16, we get $I \vdash i \wedge \phi \Rightarrow \sigma(i \Rightarrow \phi)$ is derivable in $\delta$ MNTL for any universal modality $\sigma$.

Base Case $|\sigma|=0$ :

$i \circ \phi \vdash i \Rightarrow \phi$ can be derived by basic manipulations using weakening rules and $(\vdash \Rightarrow)$. Indeed, $\phi \vdash \phi$ has a derivation in $\delta$ MNTL for any $\phi \in \operatorname{NTL}(G, H)$. The second base case can be solved as follows

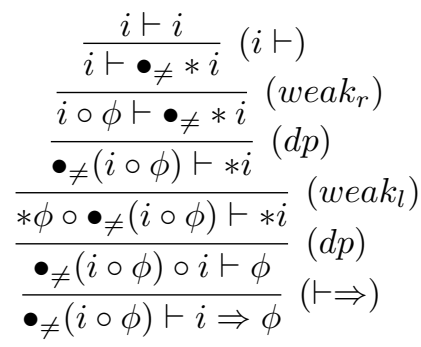

Induction Step:

As an example let us show that $i \circ \phi \vdash G \sigma(i \Rightarrow \phi)$ and $\bullet \neq(i \circ \phi) \vdash H \sigma(i \Rightarrow \phi)$ are derivable in $\delta$ MNTL assuming that $i \circ \phi \vdash \sigma(i \Rightarrow \phi)$ and $\bullet \neq(i \circ \phi) \vdash \sigma(i \Rightarrow \phi)$ are derivable in $\delta$ MNTL. In the derivations below, '(IH)' means induction hypothesis.

$$
\begin{aligned}
& \vdots(I H)
\end{aligned}
$$

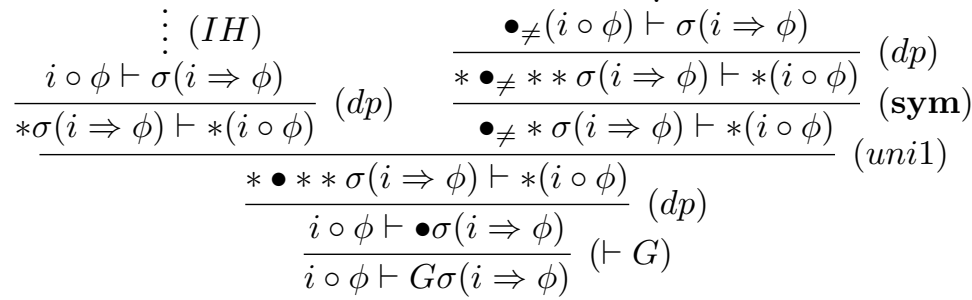

The other case is solved in the following way: 


$$
\begin{aligned}
& \vdots(I H) \quad \vdots(I H) \\
& \frac{i \circ \phi \vdash \dot{\sigma}(i \Rightarrow \phi) \quad \bullet \neq(i \circ \phi) \vdash \sigma(i \Rightarrow \phi)}{\frac{\bullet \neq \bullet \neq(i \circ \phi) \vdash \sigma(i \Rightarrow \phi)}{\bullet_{*}(\text { alio })}(d p)}
\end{aligned}
$$

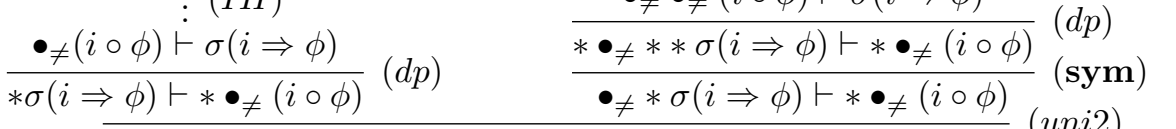

$$
\begin{aligned}
& \frac{\bullet * \sigma(i \Rightarrow \phi) \vdash * \bullet \neq(i \circ \phi)}{\bullet \neq(i \circ \phi) \vdash * \bullet * \sigma(i \Rightarrow \phi)}(d p) \\
& \bullet \neq(i \circ \phi) \vdash H \sigma(i \Rightarrow \phi) \quad(\vdash H)
\end{aligned}
$$

Although Theorem 17 is weaker than Corollary 15 (no construction of cut-free derivations), its proof relies only on the completeness of $\delta K t$ [Kra96] and on the derivability of the axiom schema $i \wedge \phi \Rightarrow \sigma(i \Rightarrow \phi)$. Unlike the completeness proof in Section 4, the proof of Theorem 17 shows how the rules (uni1), (uni2), (sym) and (alio) are essential to get completeness. This is in sharp contrast with the proof in Section 4 where the necessity of the above-mentioned rules is hidden by taking advantage of the results from [Rij92, Kra96]. The completeness proof in Section 4 also gives a weak cut-elimination theorem for $\delta$ MNTL by relying on the fact that MTL $\neq$ enjoys cut-elimination. But as we shall show in Section 6, $\delta$ MNTL does not satisfy the condition (C8), so we cannot prove cut-elimination for $\delta$ MNTL (for sequents of the general form $\mathrm{X} \vdash \mathrm{Y}$ ) directly using $(\mathrm{C} 8)$.

\section{Cut Elimination and Belnap's conditions}

A very important feature of the proof-theoretical framework DL is the existence of a very general cut-elimination theorem [Bel82]. Indeed, any display calculus satisfying Belnap's conditions (C2)-(C8) enjoys cut-elimination [Bel82]. Unfortunately $\delta$ MNTL does not satisfy (C8) recalled below; see e.g. [Wan98]:

(C8) If there are inferences $\mathcal{I}_{1}$ and $\mathcal{I}_{2}$ with respective conclusions $\mathrm{X} \vdash \phi$ and $\phi \vdash \mathrm{Y}$ with $\phi$ principal in both inferences, and if cut is applied to obtain $\mathrm{X} \vdash \mathrm{Y}$, then

- either $\mathrm{X} \vdash \mathrm{Y}$ is identical to one of $\mathrm{X} \vdash \phi$ and $\phi \vdash \mathrm{Y}$

- or there is a derivation of $\mathrm{X} \vdash \mathrm{Y}$ from the premisses of $\mathcal{I}_{1}$ and $\mathcal{I}_{2}$ in which every cut-formula of any application of cut is a proper subformula of $\phi$.

Consider the derivation,

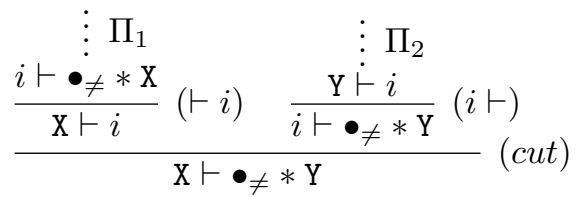

Since $i$ has no proper subformulae, $\delta$ MNTL does not satisfy (C8). However, $\delta$ MNTL enjoys a limited cut-elimination theorem by Corollary 15, and (C8) is crucial in the 
cut-elimination proofs in [Bel82, Wan98]. The situation may seem even worse since an inference of $(i \vdash)$ [resp. $(\vdash i)$ ] changes the displayed antecedent [resp. succedent] occurrence of $i$ into a succedent [resp. antecedent] part. This does not violate the condition (C4) since the occurrences of a name in some $(i \vdash)$-rule [resp. $(\vdash i)$ ] inference are not parameters: they are not substructures of some structure obtained by instantiating some structural variable.

Surprisingly, the proof of Theorem 17 shows that if $\vdash_{\text {MNTL }} \phi$, then $I \vdash \phi$ is derivable in $\delta$ MNTL minus the rule $(\vdash i)$, say $\delta^{-}$MNTL. Indeed, the $(\vdash i)$-rule is not used. Fortunately, $\delta^{-}$MNTL enjoys cut-elimination. Indeed in $\delta^{-}$MNTL, $i$ can be a succedent principal formula in an inference only in the fundamental logical axiom $i \vdash i$, and hence $\delta^{-}$MNTL obeys Belnap's (C8). Hence we can simply apply Belnap's proof giving:

Theorem 18 For every set $\mathcal{R}$ of structural rules satisfying Belnap's conditions (C2)(C8), the calculus $\delta^{-}$MNTL $+\mathcal{R}$ satisfies the conditions (C1)-(C8) and hence enjoys cut-elimination for arbitrary sequents of the form $\mathrm{X} \vdash \mathrm{Y}[\mathrm{Bel} 82]$.

Whether $\delta$ MNTL itself enjoys cut-elimination is an open question since all the derivable sequents $\mathrm{X} \vdash \mathrm{Y}$ are not necessarily of the form $I \vdash \phi$. Moreover, the above result does not guarantee that any reasonable extension of $\delta$ MNTL enjoys cut-elimination.

A preliminary version of this paper contained a strong normalisation theorem for any display calculus obtained from $\delta$ MNTL by addition of structural rules satisfying the conditions $(\mathrm{C} 2)-(\mathrm{C} 7)$. But more recent work [DG01a] has shown that the proof of strong normalisation requires more work. We have therefore relegated strong normalisation to the further work category.

\section{Pseudo Displayable Nominal Tense Logics}

Theorem 14 tells us that $\delta$ MNTL is complete for MNTL, and that it enjoys cut-elimination for sequents of the form $I \vdash \phi$. But its proof does not give us cut-elimination for arbitrary sequents $\mathrm{X} \vdash \mathrm{Y}$ in $\delta$ MNTL.

Theorem 17 tells us that $\delta$ MNTL is complete for MNTL, and its proof shows that a slightly restricted variant $\delta^{-}$MNTL is also complete for MNTL. Since $\delta^{-}$MNTL satisfies all of Belnap's conditions, it is not only complete for MNTL, but it enjoys cut-elimination for arbitrary sequents $\mathrm{X} \vdash \mathrm{Y}$. Theorem 18 then simply extends this cut-elimination result to extensions of $\delta^{-}$MNTL obtained via addition of structural rules that satisfy Belnap's conditions (C2)-(C7). By Kracht's results we know that these rules arise naturally from primitive axioms. But since our logics are defined semantically, an obvious task is to characterise these rules (or the associated primitive axioms) semantically. Without nominals, we already know the answer by Sahlqvist's theorem [Sah75].

In this section, we address this problem using techniques and results from [Bla90, Kra96] for both $\delta$ MNTL and $\delta^{-}$MNTL while preserving the cut-elimination theorems that they respectively enjoy.

Definition 19 For some class of frames $\mathcal{C}$, let $\mathcal{L}=\langle\operatorname{NTL}(H, G), \mathcal{C}\rangle$ be the nominal tense logic of $\mathcal{C}$-frames. Logic $\mathcal{L}$ is pseudo displayable if: 
(i) There is a display calculus $\delta \stackrel{\text { def }}{=} \delta$ MNTL $+\mathcal{R}$ such that $\mathcal{R}$ is a set of structural rules satisfying Belnap's conditions $(\mathrm{C} 2)-(\mathrm{C} 7)$, and

(ii) Any formula $\phi \in \operatorname{NTL}(H, G)$ is $\mathcal{L}$-valid iff $I \vdash \phi$ is derivable in $\delta$.

We need the following notion of Sahlqvist tense formula in order to study the nominal tense logics characterised by classes of frames modally definable by a Sahlqvist tense formula. We recall that a formula is positive [resp. negative] iff every propositional variable occurs under an even [resp. odd] number of negation symbols when every implication $\phi \Rightarrow \psi$ is rewritten as $\neg \phi \vee \psi$.

Definition 20 A simple Sahlqvist tense formula (see e.g. [Rij93]) from $\mathrm{TL}(H, G)$ is an implication $\phi \Rightarrow \psi$ such that:

- $\psi$ is positive

- $\phi$ is built up from negative formulae, formulae without occurrences of propositional variables, formulae of the form $\sigma \mathrm{p}$ with $\sigma$ a universal modality and $\mathrm{p} \in \operatorname{Prp}$, using only $\wedge, \vee$ and the existential modalities.

A Sahlqvist tense formula is a conjunction of formulae of the form $\sigma(\phi \Rightarrow \psi)$ with $\sigma$ a universal modality and $\phi \Rightarrow \psi$ a simple Sahlqvist tense formula.

Theorem 21 If $\phi$ is a Sahlqvist tense formula and $\vdash \stackrel{\text { def }}{=} \vdash_{\text {MNTL }}+\phi$, then any formula $\psi \in \operatorname{NTL}(H, G)$ is $\operatorname{NTL}_{\phi}$-valid iff $\vdash \psi$.

Before proving the theorem, an aside is in order.

As usual, a set $X \subseteq \operatorname{NTL}(H, G)$ is $\vdash$-consistent $\stackrel{\text { def }}{\Leftrightarrow}$ there is no finite subset $\left\{\phi_{1}, \ldots, \phi_{n}\right\} \subseteq X$ such that $\vdash \neg\left(\phi_{1} \wedge \ldots \wedge \phi_{n}\right)$. A set $X \subseteq \operatorname{NTL}(H, G)$ is called a maximal $\vdash$-consistent set $\stackrel{\text { def }}{\Leftrightarrow} X$ is $\vdash$-consistent and for all $\phi \in \operatorname{NTL}(H, G)$, either $\phi \in X$ or $\neg \phi \in X$. We write $X_{G}$ to denote the set $\{\phi \mid G \phi \in X\}$. We use the standard construction of the canonical model (see e.g. [LS77, Mak66]).

For any Sahlqvist tense formula $\phi$, if $\{\mathcal{F} \in F r \mid \mathcal{F} \models \phi\}$ contains a frame $\mathcal{F}_{1}=$ $\left(W_{1}, R_{1}\right)$ with a reflexive $w_{1} \in W_{1}$ and a frame $\mathcal{F}_{2}=\left(W_{2}, R_{2}\right)$ with an irreflexive $w_{2} \in W_{2}$ (for instance take $\phi$ to be $\mathrm{p} \Rightarrow \mathrm{p}$ ), $\mathrm{NTL}_{\phi}$ is non canonical [Bla90, Proof of Theorem 4.3.1.]. That is, there is no $\mathrm{NTL}_{\phi}$-model $\mathcal{M}=(W, R, m)$ such that for every $\vdash$-consistent set $X$ there is $w \in W$ such that for all $\psi \in X, \mathcal{M}, w \models \psi$. The arguments go as follows. $\mathcal{F}_{1} \not \models \neg(i \wedge F i)$ since $\left(W_{1}, R_{1}, m_{1}\right), w_{1} \models i \wedge F i$ with $m_{1}(i) \stackrel{\text { def }}{=}\left\{w_{1}\right\}$ and $\mathcal{F}_{2} \not \models \neg(i \wedge \neg F i)$ since $\left(W_{2}, R_{2}, m_{2}\right), w_{2} \models i \wedge \neg F i$ with $m_{2}(i) \stackrel{\text { def }}{=}\left\{w_{2}\right\}$. So neither $\neg(i \wedge F i)$ nor $\neg(i \wedge \neg F i)$ is NTL N $^{\text {-valid }}$ and neither is derivable in $\vdash_{\text {MNTL }}+\phi$ (by soundness). Thus both $(i \wedge F i)$ and $(i \wedge \neg F i)$ are $\left(\vdash_{\text {MNTL }}+\phi\right)$-consistent. But in any $\mathrm{NTL}_{\phi}$-model $\mathcal{M}, i$ is true at exactly one point, so it is impossible to have both formulae true in $\mathcal{M}$.

Proof The proof of Theorem 21 follows developments from the second completeness proof of $\vdash_{\text {MNTL }}$ in [Bla90]. Our contribution is merely to check that it also works with $\vdash_{\text {MNTL }}+\phi$ when $\phi$ is a Sahlqvist tense formula.

The canonical model for $\vdash$ is the triple $\mathcal{M}^{c} \stackrel{\text { def }}{=}\left(W^{c}, R^{c}, m^{c}\right)$ where:

$W^{c}$ is the family of all maximal $\vdash$-consistent sets 


$$
\begin{aligned}
& \text { for all } X, Y:(X, Y) \in R^{c} \stackrel{\text { def }}{\Leftrightarrow} X_{G} \subseteq Y \\
& m^{c}(\mathrm{p}) \stackrel{\text { def }}{=}\left\{X \in W^{c} \mid \mathrm{p} \in X\right\} \text { for every } \mathrm{p} \in \operatorname{Prp} \\
& m^{c}(i) \stackrel{\text { def }}{=}\left\{X \in W^{c} \mid i \in X\right\} \text { for every } i \in \text { Nom. }
\end{aligned}
$$

The usual Fundamental Theorem holds: for any $X \in W^{c}$, a formula $\psi \in X$ iff $\mathcal{M}^{c}, X \models \psi$. Since $\phi$ is a Sahlqvist tense formula, $\left(W^{c}, R^{c}\right)$ belongs to the frames of $\operatorname{NTL}_{\phi}$ (see e.g. [Sah75, Ben84, Kra96]). As in the completeness proof of $\vdash_{\text {MNTL }}$ in [Bla90], for any name $i$, there is no guarantee that $m^{c}(i)$ is a singleton. Following [Bla90, Lemma 4.3.7.], we can show that given $X \in W^{c}$, the (temporal) generated subframe $\mathcal{M}_{X}^{c} \stackrel{\text { def }}{=}\left(W_{X}^{c}, R_{X}^{c}, m_{X}^{c}\right)$ of $X$ satisfies:

for all $Y, Y^{\prime} \in W_{X}^{c}$, if some nominal $i$ belongs to $Y \cap Y^{\prime}$, then $Y=Y^{\prime}$.

We recall that for any $Y \in W^{c}: Y \in W_{X}^{c} \stackrel{\text { def }}{\Leftrightarrow}$ there is a finite sequence $\left\langle Y_{0}, \ldots, Y_{l}\right\rangle$ such that $\left(R^{c}\right)^{-1}$ is the converse of $R^{c}$ and:

$$
Y_{0}=X \text { and } Y_{l}=Y \text { and for any } j \in\{0, \ldots, l-1\},\left(Y_{j}, Y_{j+1}\right) \in R^{c} \cup\left(R^{c}\right)^{-1} \text {. }
$$

Moreover, $\mathcal{M}_{X}^{c}$ is a frame for $\mathrm{NTL}_{\phi}$. Indeed, $\left(W^{c}, R^{c}\right) \models \phi$ and by [Bla90, Corollary 3.2.1], $\left(W_{X}^{c}, R_{X}^{c}\right) \models \phi$ (see also [GT75]). By [Bla90, Theorem 3.2.1], for any $\psi \in X: \mathcal{M}_{X}^{c}, X \models \psi$ iff $\mathcal{M}^{c}, X \models \psi$.

Our proof is almost finished since $m_{X}^{c}$ behaves better than $m^{c}$ but $m_{X}^{c}(i)$ may be the empty set for some name $i$. Let $S$ be the set

$$
S \stackrel{\text { def }}{=}\left\{i \in \operatorname{Nom} \mid m_{X}^{c}(i)=\emptyset\right\}
$$

Let $\mathcal{M} \stackrel{\text { def }}{=}(W, R, m)$ be the model such that:

$$
\begin{aligned}
& W \stackrel{\text { def }}{=}\{1,2\} \times W_{X}^{c} \\
& \left(\left\langle j_{1}, Y_{1}\right\rangle,\left\langle j_{2}, Y_{2}\right\rangle\right) \in R \stackrel{\text { def }}{\Leftrightarrow} j_{1}=j_{2} \text { and }\left(Y_{1}, Y_{2}\right) \in R_{X}^{c} \\
& m(\mathrm{p}) \stackrel{\text { def }}{=}\{\langle j, Y\rangle \in W \mid \mathrm{p} \in Y, 1 \leq j \leq 2\} \text { for any } \mathrm{p} \in \operatorname{Prp} \\
& m(i) \stackrel{\text { def }}{=}\{\langle 1, Y\rangle\} \text { for any } i \in \text { Nom } \backslash S \text { with } m_{X}^{c}(i)=\{Y\} \\
& m(i) \stackrel{\text { def }}{=}\{\langle 2, X\rangle\} \text { for any } i \in S .
\end{aligned}
$$

A similar construction is used in the proof of Lemma 9. The structure $\mathcal{M}$ is a disjoint union of $\mathcal{M}_{X}^{c}$ with itself, except for the restriction of the valuation function to nominals, and therefore $(W, R)$ is a frame of $\mathrm{NTL}_{\phi}$. Indeed, every class of frames definable by a formula $\phi \in \mathrm{TL}(H, G)$ is closed under disjoint union; see e.g. [Bla90, Section 3.2.2] or [GT75]. Moreover, $m$ is a correct valuation and for any $\psi \in X$ : $\mathcal{M},\langle 1, X\rangle \models \psi$ iff $\mathcal{M}_{X}^{c}, X \models \psi$.

If instead of nominals, we allow the difference operator $[\neq]$ in the language, things get worse. Indeed, the constraints relative to the nominals affect only the valuation function, whereas with the difference operator the constraints affect the relationships between relations. This partly explains why the powerful irreflexivity rule is often needed (see e.g. [Ven93, Rij92, Bal99]).

Recall that a primitive formula is of the form $\phi \Rightarrow \psi$ where both $\phi$ and $\psi$ are built from propositional variables from $\operatorname{Prp}$ and $T$ with the help of $\wedge, \vee, F, P$ and $\langle\neq\rangle$ only, and $\phi$ contains each propositional variable at most once [Kra96]. 
Theorem 22 For some class of frames $\mathcal{C}$, let $\mathcal{L}=\langle\operatorname{NTL}(H, G), \mathcal{C}\rangle$ be the nominal tense logic of $\mathcal{C}$-frames, and $\phi$ be a conjunction of primitive axioms in $\operatorname{TL}(G, H)$ such that $\mathcal{C}=\{\mathcal{F} \in F r: \mathcal{F} \models \phi\}$. Then, $\mathcal{L}$ is pseudo displayable.

Proof Using the effective procedure from [Kra96, Section 5], to each primitive axiom $\phi^{\prime}$, conjunct of $\phi$, we associate a finite set of structural rules. This provides a constructive way to find a set $\mathcal{R}$ of structural rules such that for any $\psi \in \operatorname{NTL}(H, G)$ with $\vdash \stackrel{\text { def }}{=} \vdash_{\text {MNTL }}+\phi: \vdash \psi$ iff $I \vdash \psi$ is derivable in $\delta$ MNTL $+\mathcal{R}$. By Theorem 21, $\vdash$ axiomatises $\mathcal{L}$ since every primitive formula in $\operatorname{TL}(G, H)$ is a Sahlqvist tense formula and therefore $\mathcal{L}$ is pseudo displayable.

Note that for soundness and completeness, the proof of Theorem 22 only requires the calculus $\delta^{-}$MNTL $+\mathcal{R}$. Moreover, by Theorem 18, $\delta^{-}$MNTL $+\mathcal{R}$ satisfies Belnap's conditions and enjoys cut-elimination for arbitrary sequents. As a consequence, for every $\psi \in \operatorname{NTL}(H, G), I \vdash \psi$ is derivable in $\delta$ MNTL $+\mathcal{R}$ iff $I \vdash \psi$ has a cut-free derivation in $\delta$ MNTL $+\mathcal{R}$.

Corollary 23 Let $\mathcal{L}$ be a logic defined in Theorem 22. Then, every formula $\phi \in$ $\operatorname{NTL}(H, G)$ is $\mathcal{L}$-valid iff $I \vdash \phi$ is derivable in $\delta^{-}$MNTL $+\mathcal{R}$, where $\mathcal{R}$ is the set of structural rules defined in the proof of Theorem 22 .

Another class of pseudo displayable nominal tense logics can be identified by considering the first completeness proof of $\delta$ MNTL from Section 4 .

Theorem 24 For some class of frames $\mathcal{C}$ which is closed under disjoint unions and isomorphic copies, let $\mathcal{L}=\langle\operatorname{NTL}(H, G), \mathcal{C}\rangle$ be the nominal tense logic of $\mathcal{C}$-frames, let $\mathcal{L}_{\neq}=\langle\mathrm{TL}(H, G,[\neq]), \mathcal{C}\rangle$ be the tense logic of inequality of $\mathcal{C}$-frames, and let $\gamma$ be a conjunction of primitive axioms over the language $\operatorname{TL}(H, G,[\neq])$ such that $\vdash_{\neq}+\gamma$ axiomatises $\mathcal{L}_{\neq}$. Then, $\mathcal{L}$ is pseudo displayable.

The careful reader might observe that the irreflexivity rule is not present in $\vdash_{\neq}+\gamma$. Moreover, unlike Theorem 22, the primitive axioms in Theorem 24 are built from the language that does admit $[\neq]$.

Proof (sketch) By [Kra96, Lemma 13], $\vdash_{\neq}+\gamma$ is pseudo displayable in the sense defined in [Kra96]. Let $\delta \mathrm{MTL}_{\neq}+\mathcal{R}_{\gamma}$ be the display calculus for $\vdash_{\neq}+\gamma$ with $\mathcal{R}_{\gamma}$ the set of structural rules obtained from $\gamma$ using the effective procedure of [Kra96, Section 5]. The proof of Theorem 14 can be adapted to prove that $\delta$ MNTL $+\mathcal{R}_{\gamma}$ pseudo displays $\mathcal{L}=\langle\operatorname{NTL}(H, G), \mathcal{C}\rangle$.

Indeed one can show that if $\phi$ is $\mathcal{L}$-valid, then $a(\phi)$ is $\mathcal{L}_{\neq \text {-valid and therefore } I \vdash}$ $a(\phi)$ has a cut-free derivation in $\delta \mathrm{MTL}_{\neq}+\mathcal{R}_{\gamma}$. By adapting the proof of Theorem 14, we can obtain that $I \vdash \phi$ has a cut-free derivation in $\delta$ MNTL $+\mathcal{R}_{\gamma}$. Observe that any formula $\psi \in \operatorname{NTL}(H, G)$ is $\mathcal{L}$-valid iff $a(\psi)$ is $\mathcal{L}_{\neq}$-valid since $\mathcal{C}$ is closed under disjoint unions and isomorphic copies (see Lemma 9). For the soundness part, we show that if $I \vdash \phi$ is derivable in $\delta$ MNTL $+\mathcal{R}_{\gamma}$, then $\phi$ is $\mathcal{L}$-valid. To do so, we prove that if $\mathrm{X} \vdash \mathrm{Y}$ is derivable in $\delta$ MNTL $+\mathcal{R}_{\gamma}$, then $\varphi_{\{\mathrm{X}, \mathrm{Y}\}} \Rightarrow(a(\mathrm{X}) \Rightarrow c(\mathrm{X}))$ is $\mathcal{L}_{\neq- \text {-valid. We use }}$ the two lemmas below: 
1. Let $r$ be an inference rule in $\delta$ MNTL $+\mathcal{R}_{\gamma}$ such that for any inference

$$
\frac{\mathrm{X}_{1} \vdash \mathrm{Y}_{1} \ldots \mathrm{X}_{n} \vdash \mathrm{Y}_{n}}{\mathrm{X}_{n+1} \vdash \mathrm{Y}_{n+1}}(r)(n \geq 0)
$$

if for $i \in\{1, \ldots, n\}, a\left(\mathrm{X}_{i}\right) \Rightarrow c\left(\mathrm{Y}_{i}\right)$ is $\mathcal{L}_{\neq^{-}}$valid, then $a\left(\mathrm{X}_{n+1}\right) \Rightarrow c\left(\mathrm{Y}_{n+1}\right)$ is $\mathcal{L}_{\neq^{-}}$ valid. Then, if for $i \in\{1, \ldots, n\}, \varphi_{\left\{\mathrm{x}_{i}, \mathrm{Y}_{i}\right\}} \Rightarrow\left(a\left(\mathrm{X}_{i}\right) \Rightarrow c\left(\mathrm{Y}_{i}\right)\right)$ is $\mathcal{L}_{\neq^{-}}$-valid, then $\varphi_{\left\{\mathrm{x}_{n+1}, \mathrm{Y}_{n+1}\right\}} \Rightarrow\left(a\left(\mathrm{X}_{n+1}\right) \Rightarrow s\left(\mathrm{Y}_{n+1}\right)\right)$ is $\mathcal{L}_{\neq}$-valid.

2. For all the rules $r$ in $\delta$ MNTL $+\mathcal{R}_{\gamma}$ except the $(\vdash i)$-rule, for any inference

$$
\frac{\mathrm{X}_{1} \vdash \mathrm{Y}_{1} \ldots \mathrm{X}_{n} \vdash \mathrm{Y}_{n}}{\mathrm{X}_{n+1} \vdash \mathrm{Y}_{n+1}}(r)(n \geq 0)
$$

if for $1 \leq i \leq n, a\left(\mathrm{X}_{i}\right) \Rightarrow c\left(\mathrm{Y}_{i}\right)$ is $\mathcal{L}_{\neq^{-}}$valid, then so is $a\left(\mathrm{X}_{n+1}\right) \Rightarrow c\left(\mathrm{Y}_{n+1}\right)$.

Proof of (1): For $i \in\{1, \ldots, n+1\}$, there is $\phi_{i} \in \operatorname{NTL}(H, G)$ such that $a\left(\phi_{i}\right)=$ $a\left(\mathrm{X}_{i}\right) \Rightarrow c\left(\mathrm{Y}_{i}\right)$ and $\varphi_{\left\{\phi_{i}\right\}}=\varphi_{\left\{\mathrm{x}_{i}, \mathrm{Y}_{i}\right\}}$. So, for $i \in\{1, \ldots, n+1\}, \varphi_{\left\{\mathrm{x}_{i}, \mathrm{Y}_{i}\right\}} \Rightarrow$

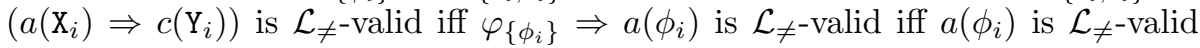
(by Lemma 8 and Lemma 9) iff $a\left(\mathrm{X}_{i}\right) \Rightarrow c\left(\mathrm{Y}_{i}\right)$ is $\mathcal{L}_{\neq \text {-valid. This suffices to get }}$ the desired result.

Proof of (2): The proof follows from [Kra96] and by observing that $\phi \Rightarrow(\mathrm{p} \wedge[\neq] \neg \mathrm{p})$

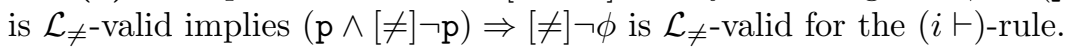

Let us conclude the soundness proof. By (1) and (2), for any inference rule $r$ in $\delta$ MNTL $+\mathcal{R}_{\gamma}$ minus $(\vdash i)$, for any inference

$$
\frac{\mathrm{X}_{1} \vdash \mathrm{Y}_{1} \ldots \mathrm{X}_{n} \vdash \mathrm{Y}_{n}}{\mathrm{X}_{n+1} \vdash \mathrm{Y}_{n+1}}(r)(n \geq 0)
$$

if for $1 \leq i \leq n, \varphi_{\left\{\mathrm{x}_{i}, \mathrm{Y}_{i}\right\}} \Rightarrow\left(a\left(\mathrm{X}_{i}\right) \Rightarrow c\left(\mathrm{Y}_{i}\right)\right)$ is $\mathcal{L}_{\neq^{-}}$valid, then so is $\varphi_{\left\{\mathrm{x}_{n+1}, \mathrm{Y}_{n+1}\right\}} \Rightarrow$ $\left(a\left(\mathrm{X}_{n+1}\right) \Rightarrow s\left(\mathrm{Y}_{n+1}\right)\right)$. The proof of Theorem 10 gives a similar property for $(i \vdash)$. By Lemma 9: $I \vdash \phi$ is derivable in $\delta$ MNTL $+\mathcal{R}_{\gamma}$ implies $\phi$ is $\mathcal{L}$-valid.

As a consequence of Theorem 24:

Corollary 25 For every $\psi \in \operatorname{NTL}(H, G), I \vdash \psi$ is derivable in $\delta$ MNTL $+\mathcal{R}_{\gamma}$ iff $I \vdash \psi$ has a cut-free derivation in $\delta$ MNTL $+\mathcal{R}_{\gamma}$.

Unlike the proof of Theorem 22, the proof of Theorem 24 does not allow us to deduce an analogous completeness result for primitive extensions of $\delta^{-}$MNTL since the latter is based upon the proof of Theorem 14 where we explicitly need to use both introduction rules for nominals.

\section{Concluding Remarks}

We have defined display calculi for a large class of nominal tense logics. All our calculi enjoy cut-elimination for restricted sequents of the form $I \vdash \phi$. A simple observation on the use of the rules for introducing nominals shows that a slight variant of all our calculi also enjoy cut-elimination for arbitrary sequents $\mathrm{X} \vdash \mathrm{Y}$.

The following open problems are worth investigating: 
1. How to extend the completeness result for primitive extensions of $\delta$ MNTL to primitive extensions of $\delta^{-}$MNTL for the class of nominal tense logics from Theorem $24 ?$

2. How to define structural rules in DL from axioms containing names?

3. We have proposed a way to formalise proof systems for nominal tense logics within DL. Numerous nominal tense logics are known to be decidable (see e.g. [Bla90, Bla93, PT91]). How to design uniform decision procedures based on our calculi?

4. Theorem 22 and Theorem 24 establish two classes of pseudo displayable nominal tense logics. Are they really different classes?

5. A possible answer to (1) is to characterise the class of Sahlqvist tense formulae $\phi$ such that $\vdash_{\neq}+\phi$ axiomatises $\langle\operatorname{TL}(H,[\neq], G),\{\mathcal{F} \mid \mathcal{F}=\phi\}\rangle$. This is roughly equivalent to determining when the irreflexivity rule is superfluous (see e.g. [Ven93]). A partial answer can be found in [Bal99].

Acknowledgements We thank Jeremy E Dawson for helpful comments on an earlier version of this paper. The first author's visit to A.R.P. was supported by an Australian Research Council International Fellowship. He is currently on leave from Laboratoire LEIBNIZ, Grenoble, France. The second author is supported by an Australian Research Council Queen Elizabeth II Fellowship.

\section{References}

[ABM00] C. Areces, P. Blackburn, and M. Marx. Complexity of hybrid temporal logics. Journal of the IGPL, 8(5):653-679, 2000.

[Are00] C. Areces. Logic Engineering: The Case of Description and Hybrid Logics. PhD thesis, University of Amsterdam, 2000.

[Bal99] Ph. Balbiani. Inequality without irreflexivity. Journal of Applied Non Classical Logics, 9(4), 1999.

[BD97] Ph. Balbiani and S. Demri. Prefixed tableaux systems for modal logics with enriched languages. In IJCAI-15, pages 190-195. Morgan Kaufmann, 1997.

[Bel82] N. Belnap. Display logic. Journal of Philosophical Logic, 11:375-417, 1982.

[Ben84] J. van Benthem. Correspondence Theory. In D. Gabbay and F. Günthner, editors, Handbook of Philosophical Logic, Volume II, pages 167-247. Reidel, Dordrecht, 1984.

[Bla90] P. Blackburn. Nominal Tense Logic and Other Sorted Intensional Frameworks. PhD thesis, Univ. of Edinburgh, 1990.

[Bla93] P. Blackburn. Nominal tense logic. NDJFL, 34(1):56-83, 1993.

[Bla00] P. Blackburn. Internalizing labelled deduction. Journal of Logic and Computation, 10(1):137-168, 2000

[Dem96] S. Demri. A simple tableau system for the logic of elsewhere. In P. Miglioli, U. Moscato, D. Mundici, and M. Ornaghi, editors, TABLEAUX'96, pages 177192. LNAI 1071, Springer-Verlag, 1996. 
[Dem99] S. Demri. Sequent calculi for nominal tense logics: a step towards mechanization? In N. Murray, editor, TABLEAUX'99, pages 140-154. LNAI 1617, SpringerVerlag, 1999.

[DG98] J. Dawson and R. Goré. A mechanised proof system for relation algebra using display logic. In J. Dix, L. Fariñas del Cerro, and U. Furbach, editors, JELIA'98, pages 264-278. LNAI 1489, Springer-Verlag, 1998.

[DG99] S. Demri and R. Goré. Cut-free display calculi for nominal tense logics. In N. Murray, editor, TABLEAUX'99, pages 155-170. LNAI 1617, Springer-Verlag, 1999.

[DG00a] S. Demri and R. Goré. Display calculi for logics with relative accessibility relations. Journal of Logic, Language and Information, 9:213-236, 2000.

[DG00b] S. Demri and R. Goré. An $\mathrm{O}\left((n . \log n)^{3}\right)$-time transformation from Grz into decidable fragments of classical first-order logic. In R. Caferra and G. Salzer, editors, Automated Deduction in Classical and Non Classical Logics, pages 153167. LNAI 1761, Springer-Verlag, 2000.

[DG01a] J. Dawson and R. Goré. Mechanising cut elimination for display calculi. Technical Report TR-CSL-01-02, A.R.P., A.N.U., 2001. Accessible via http://arp.anu . edu.au/ on WWW.

[DG01b] S. Demri and R. Goré. Theoremhood preserving maps characterising cut elimination for modal provability logics. Journal of Logic and Computation, 2001. to appear.

[dM94] M. d'Agostino and M. Mondadori. The taming of the cut. Classical refutations with analytic cut. Journal of Logic and Computation, 4(3):285-319, 1994.

[Gab81] D. Gabbay. An irreflexivity lemma with applications to axiomatization of conditions on tense frames. In U. Mönnich, editor, Aspects of Philosophical Logic, pages 67-89. Reidel, 1981.

[Gab96] D. Gabbay. Labelled Deductive Systems. Oxford University Press, 1996.

[Gar86] G. Gargov. Two completeness theorems in the logic for data analysis. Technical Report 581, Institute of Computer Science, Polish Academy of Sciences, Warsaw, 1986.

[GG93] G. Gargov and V. Goranko. Modal logic with names. Journal of Philosophical Logic, 22(6):607-636, 1993.

[Gor90] V. Goranko. Modal definability in enriched languages. Notre Dame Journal of Formal Logic, 31:81-105, 1990.

[Gor95] R. Goré. Intuitionistic logic redisplayed. Technical Report TR-ARP-1-95, A.R.P., A.N.U., 1995. Accessible via http://arp.anu.edu.au/ on WWW.

[Gor96a] V. Goranko. Hierarchies of modal and temporal logics with references pointers. Journal of Logic, Language and Information, 5:1-24, 1996.

[Gor96b] R. Goré. On the completeness of classical modal display logic. In H. Wansing, editor, Proof theory of modal logic, pages 137-140. Kluwer, 1996.

[Gor97] R Goré. Cut-free display calculi for relation algebras. In D. van Dalen and M. Bezem, editors, CSL96, pages 198-210. Springer-Verlag, LNCS 1258, 1997.

[Gor98a] R. Goré. Gaggles, Gentzen and Galois: Cut-free display calculi for algebraizable logics. Logic Journal of the IGPL, 6(5):669-694, 1998.

[Gor98b] R. Goré. Substructural logics on display. Logic Journal of the IGPL, 6(3):451-504, 1998. 
[GT75] R. Goldblatt and S. Thomason. Axiomatic classes in propositional modal logic. In J. Crossley, editor, Algebra and Logic, pages 163-173. Springer-Verlag, LNM 450, 1975.

[Kon97a] B. Konikowska. A logic for reasoning about relative similarity. Studia Logica, 58(1):185-226, 1997.

[Kon97b] B. Konikowska. A logic for reasoning about similarity. In [Oe97], pages 462-491, 1997.

[Koy92] R. Koymans. Specifying message passing and time-critical systems with temporal logic. LNCS 651, Springer-Verlag, 1992.

[Kra96] M. Kracht. Power and weakness of the modal display calculus. In H. Wansing, editor, Proof theory of modal logic, pages 93-121. Kluwer, 1996.

[LS77] E. J. Lemmon and D. Scott. An introduction to modal logic. American Philosophical Quarterly, 1977.

[Mak66] D. Makinson. On some completeness theorems in modal logic. The Journal of Symbolic Logic, 12:379-384, 1966.

[Oe97] E. Orłowska (ed.). Incomplete Information: Rough Set Analysis. Studies in Fuzziness and Soft Computing. Physica Verlag, Heidelberg, 1997.

[Orło84] E. Orłowska. Logic of indiscernibility relations. In A. Skowron, editor, 5th Symposium on Computation Theory, pages 177-186. LNCS 208, Springer-Verlag, 1984.

[Orło88] E. Orłowska. Relational interpretation of modal logics. In H. Andréka, D. Monk, and I. Németi, editors, Algebraic logic. Colloquia Mathematica Societatis Janos Bolyai 54, pages 443-471, Amsterdam, 1988. North Holland.

[Orło91] E. Orłowska. Relational proof systems for some AI logics. In Ph. Jorrand and J. Kelemen, editors, FAIR'91, pages 33-47. Springer-Verlag, LNAI 535, 1991.

[Orło92] E. Orłowska. Relational proof system for relevant logics. The Journal of Symbolic Logic, 57(4):1425-1440, December 1992.

[Paw81] Z. Pawlak. Information systems theoretical foundations. Information Systems, 6(3):205-218, 1981.

[PT85] S. Passy and T. Tinchev. PDL with data constants. Information Processing Letters, 20:35-41, 1985.

[PT91] S. Passy and T. Tinchev. An essay in combinatory dynamic logic. Information and Computation, 93:263-332, 1991.

[Res98] G. Restall. Displaying and deciding substructural logics 1: logics with contraposition. Journal of Philosophical Logic, 27(2):179-216, 1998.

[Rij92] M. de Rijke. The modal logic of inequality. The Journal of Symbolic Logic, 57(2):566-584, 1992.

[Rij93] M. de Rijke. Extending modal logic. PhD thesis, ILLC, Amsterdam, 1993.

[Sah75] H. Sahlqvist. Completeness and correspondence in the first and second order semantics for modal logics. In S. Kanger, editor, 3rd Scandinavian Logic Symposium, pages 110-143. North Holland, 1975.

[Sai88] I. Sain. Is 'some-other-time' sometimes better than 'sometime' for proving partial correctness of programs. Studia Logica, 47(3):278-301, 1988.

[Seg81] K. Segerberg. A note on the logic of elsewhere. Theoria, 47:183-187, 1981.

[Sel97] J. Seligman. The logic of correct description. In M. de Rijke, editor, Advances in intensional logic, pages 107-135. Applied Logic Series, Kluwer, 1997. 
[Tza99] M. Tzakova. Tableau calculi for hybrid logics. In N. Murray, editor, TABLEAUX'99, pages 278-292. LNAI 1617, Springer-Verlag, 1999.

[Ven93] Y. Venema. Derivation rules as anti-axioms in modal logic. The Journal of Symbolic Logic, 58(3):1003-1034, 1993.

[Wan94] H. Wansing. Sequent calculi for normal modal propositional logics. Journal of Logic and Computation, 4(2):125-142, 1994.

[Wan98] H. Wansing. Displaying Modal Logic, volume 3 of Trends in Logic. Kluwer Academic Publishers, Dordrecht, 1998. 